\title{
Time-Consistent and Market-Consistent Evaluations
}

\author{
Mitja Stadje \\ Tilburg University, CentER \& Netspar \\ Dept. of Econometrics and Operations Research \\ P.O. Box 90153 \\ 5000 LE Tilburg \\ The Netherlands \\ Email: m.a.stadje@uvt.nl \\ Antoon Pelsser* \\ Maastricht University \& Netspar \\ Dept. of Quantitative Economics and Dept. of Finance \\ P.O. Box 616 \\ 6200 MD Maastricht \\ The Netherlands \\ Email: a.pelsser@maastrichtuniversity.nl
}

First version: April 15, 2010

This version: November 5, 2018

\begin{abstract}
We consider evaluation methods for payoffs with an inherent financial risk as encountered for instance for portfolios held by pension funds and insurance companies. Pricing such payoffs in a way consistent to market prices typically involves combining actuarial techniques with methods from mathematical finance. We propose to extend standard actuarial principles by a new market-consistent evaluation procedure which we call 'two step market evaluation.' This procedure preserves the structure of standard evaluation techniques and has many other appealing properties. We give a complete axiomatic characterization for two step market evaluations. We show further that in a dynamic setting with continuous stock prices every evaluation which is time-consistent and market-consistent is a two step market evaluation. We also give characterization results and examples in terms of $g$-expectations in a Brownian-Poisson setting.
\end{abstract}

Keywords: Actuarial valuation principles, financial risk, market-consistency, time-consistency.

\footnotetext{
${ }^{*}$ The authors would like to thank Damir Filipovic, the participants of the AFMath2010 conference, the DGVFM 2010 Scientific Day, and seminar participants at the Institut Henri Poincaré for useful comments and suggestions on an earlier version of this paper. We also want to thank the referee for constructive suggestions which led to several improvements of the paper.
} 


\section{Introduction}

We investigate evaluation methods for payoffs with an inherent financial risk and propose a new market-consistent procedure to evaluate these payoffs. Our procedure yields the extension of many standard actuarial principles into both time-consistent and market-consistent directions.

Many insurance companies sell products which depend on financial as well as non-financial risk. Typical examples are equity-linked insurance contracts or catastrophe insurance futures and bonds. Pricing such payoffs in a way consistent to market prices usually involves combining actuarial techniques with methods from mathematical finance. The minimal conditions which any market-consistent evaluation should satisfy is that a purely financial replicable payoff should be equal to the amount necessary to replicate it.

Standard actuarial premium principles are typically based on a pooling argument which justifies applying the law of large numbers to price using the expectation with respect to the physical measure and then to take an additional risk load. With these principles one usually considers a static premium calculation problem: what is the price today of an insurance contract with payoff at time T? See for example the textbooks by Bühlmann (1970), Gerber (1979), or Kaas et al. (2008). Also, the concept of convex risk measures and the closely related one of monetary utility functions have been studied in such a static setting. See for example Föllmer and Schied (2002), Frittelli and Rosazza Gianin (2002), Jouini et al. (2008), and Filipovic and Kupper (2008).

In financial pricing one usually considers a "dynamic" pricing problem: how does the price evolve over time until the final payoff date $T$ ? This dynamic perspective is driven by the focus on hedging and replication. This literature started with the seminal paper of Black and Scholes (1973) and has been immensely generalized to broad classes of securities and stochastic processes; see Delbaen and Schachermayer (1994).

In recent years, researchers have begun to investigate risk measures in a dynamic setting, the central question being the construction of time-consistent ("dynamic") risk measures. See Riedel (2004), Roorda et al. (2005), Cheridito et al. (2006), Rosazza Gianin (2006), Artzner et al. (2007). In a dynamical context time-consistency is a natural approach to glue together static evaluations. It means that the same value is assigned to a financial position regardless of whether it is calculated over two time periods at once or in two steps backwards in time. In a recent paper Jobert and Rogers (2008) show how time-consistent valuations can be constructed via backward induction of static one-period risk-measures (or "valuations"). See also Hardy and Wirch (2005) for an example with the risk measure given by Average Value at Risk.

An important branch of literature considers risk measures/valuations in a so-called marketconsistent setting. This started with the pricing of contracts in an incomplete-market setting, where one seeks to extend the arbitrage-free pricing operators (which are only defined in a complete-market setting) to the larger space of (partially) unhedgeable contracts. One approach to evaluate the payoff in this situation is by utility indifference pricing: the investor pays the amount such that he is no worse off in expected utility terms than he would have been without the claim. The paper by Hodges and Neuberger (1989) is often cited for the root-idea of this stream of literature. For other contributions in this direction, see for instance Henderson (2002), Young and Zariphopoulou (2002), Hobson (2004), Musiela and Zariphopoulou (2004a), Monoyios (2006), and the recent book by Carmona (2009).

Several papers deal with the extension of the arbitrage-free pricing operators using (local) risk-minimisation techniques and the related notion of minimal martingale measures; see 
Föllmer and Schweizer (1989), Schweizer (1995), Delbaen and Schachermayer (1996). A rich duality theory has been developed that establishes deep connections between utility maximisation and minimisation over martingale measures; see Cvitanic and Karatzas (1992), Kramkov and Schachermayer (1999). A very elegant summary is given by Rogers (2001). Another stream of the literature, where the class of martingale measures considered is restricted, is given by the works on good-deal bound pricing, see Cochrane and Saá-Requejo (2000), Černý and Hodges (2002), and Björk and Slinko (2006).

Using utility-indifference (and duality) methods, the market-consistency of pricing operators is automatically induced. However, an explicit formal definition of market-consistent pricing operators has only begun to emerge recently; see Kupper et al. (2008), Malamud et al. (2008), Barrieu and El Karoui (2005, 2009), and Knispel et al. (2011).

In this paper we investigate well-known actuarial premium principles such as the variance principle and the standard-deviation principle, and study their extension into both timeconsistent and market-consistent directions. To do this, we introduce the concept of two step market evaluations and study their properties. Two step market evaluations convert any evaluation principle into a market-consistent one by applying the actuarial principle to the residual risk which remains after having conditioned on the future development of the stock price. This operator splitting preserves the structure and the computationally tractability of the original actuarial evaluation. Furthermore, we get some appealing properties like numeraire invariance. We are able to give a complete axiomatic characterization for two step market evaluations and show that these axioms are satisfied in a setting where the stock process is continuous and the insurance process is revealed at fixed time instances (or more generally has predictable jumps). This provides a strong argument for the use of two step market evaluations. We also consider some time-consistent extensions of our market-consistent evaluations to continuous time in a Brownian-Poisson setting. For this we need some results from the theory of backward stochastic differential equations (BSDEs), also called $g$-expectations. For background material on BSDEs we refer to El Karoui et al. (1997).

The paper is organized as follows. In Section 2 we define conditional evaluations, give some background material, and recall some of the most standard actuarial principles. In Section 3 the notion of market-consistency is defined and two step market evaluations are introduced and motivated. We give a complete axiomatic characterization for two step market evaluations. In Section 4 it is shown that in a dynamic setting with continuous stock prices every evaluation which is time-consistent and market-consistent can be viewed as a two step market evaluation. In Section 5 we extend our evaluations to a continuous-time setup with processes with jumps. Section 6 gives a summary and conclusions. Section 7 contains the proofs of our results.

\section{Conditional Evaluations}

Let $(\Omega, \mathcal{F}, \mathbb{P})$ be a probability space. Equalities and inequalities between random variables are understood in the $\mathbb{P}$-almost sure sense unless explicitly stated otherwise. The space of bounded random variables will be denoted by $L^{\infty}(\Omega, \mathcal{F}, \mathbb{P})\left(L^{\infty}(\mathcal{F})\right.$ for short). The space of bounded, non-negative random variables will be denoted by $L_{+}^{\infty}(\mathcal{F})$. The space of random variables which are integrable with respect to $\mathbb{P}$ will be denoted by $L^{1}(\Omega, \mathcal{F}, \mathbb{P})\left(L^{1}(\mathcal{F})\right.$ for short). Financial and insurance positions are represented by random variables $H \in L^{\infty}(\mathcal{F})$ where $H(\omega)$ is the discounted net loss of the position at maturity under the scenario $\omega$. Now given a $\sigma$-algebra $\mathcal{G} \subset \mathcal{F}$, with information available to the agent, we can define a conditional evaluation: 
Definition 2.1 A mapping $\Pi_{\mathcal{G}}: L^{\infty}(\mathcal{F}) \rightarrow L^{\infty}(\mathcal{G})$ is called a $\mathcal{G}$-conditional evaluation if the following axioms hold:

- Normalization: $\Pi_{\mathcal{G}}(0)=0$.

- $\mathcal{G}$-Cash Invariance: $\Pi_{\mathcal{G}}(H+m)=\Pi_{\mathcal{G}}(H)+m$ for $H \in L^{\infty}(\mathcal{F})$ and $m \in L^{\infty}(\mathcal{G})$.

- $\mathcal{G}$-Convexity: For $H_{1}, H_{2} \in L^{\infty}(\mathcal{F}) \Pi_{\mathcal{G}}\left(\lambda H_{1}+(1-\lambda) H_{2}\right) \leq \lambda \Pi_{\mathcal{G}}\left(H_{1}\right)+(1-\lambda) \Pi_{\mathcal{G}}\left(H_{2}\right)$ for all $\lambda \in L^{\infty}(\mathcal{G})$ with $0 \leq \lambda \leq 1$.

- $\mathcal{G}$-Local Property: $\Pi_{\mathcal{G}}\left(I_{A} H_{1}+I_{A^{c}} H_{2}\right)=I_{A} \Pi_{\mathcal{G}}\left(H_{1}\right)+I_{A^{c}} \Pi_{\mathcal{G}}\left(H_{2}\right)$ for all $H \in L^{\infty}(\mathcal{F})$ and $A \in \mathcal{G}$.

- Fatou property: For any bounded sequence $\left(H_{n}\right)$ which converges a.s. to $H$

$$
\Pi_{\mathcal{G}}(H) \leq \liminf _{n} \Pi_{\mathcal{G}}\left(H_{n}\right)
$$

Normalization guarantees that the null position does not require any capital reserves. If $\Pi$ is not normal then the agent can consider the operator $\Pi(H)-\Pi(0)$ without changing his preferences. Convexity, which under cash invariance is equivalent to quasiconvexity, says that diversification should not be penalized. Cash invariance gives the interpretation of $\Pi(H)$ as a capital reserve. The local property is motivated in the following way. Since the agent has the information given by $\mathcal{G}$ he knows if the event $A$ has happened or not and should adjust his evaluation accordingly. If $\Pi$ satisfies

- Monotonicity: For $H_{1}, H_{2} \in L^{\infty}(\mathcal{F})$ with $H_{1} \leq H_{2} \Pi_{\mathcal{G}}\left(H_{1}\right) \leq \Pi_{\mathcal{G}}\left(H_{2}\right)$

then we will call $\Pi$ a monotone conditional evaluation. Monotonicity postulates that if in a.s. any scenario $H_{2}$ causes a greater higher loss than $H_{1}$ then the premium charged for $\mathrm{H}_{2}$ (or the capital reserve held) should be greater than for $H_{1}$. Note that if $\Pi_{\mathcal{G}}$ is monotone then $\rho_{\mathcal{G}}(H):=\Pi_{\mathcal{G}}(-H)$ defines a conditional convex risk measure and $U_{\mathcal{G}}(H):=-\rho_{\mathcal{G}}(H)$ defines a conditional monetary utility function. For the definition of a convex risk measure, see Föllmer and Schied (2002), or Frittelli and Rosazza Gianin (2002). In particular, all results in this paper also hold (with obvious change of signs) for conditional convex risk measures and conditional monetary utility functions.

Remark 2.2 It has been shown in Cheridito et al. (2006) that the local property must be satisfied if $\Pi_{\mathcal{G}}$ is monotone and cash invariant. Moreover, the local property is also implied by convexity. Indeed, if $\Pi_{\mathcal{G}}$ is convex, then we have for $A \in \mathcal{G}$ that clearly $\Pi_{\mathcal{G}}\left(1_{A} H_{1}+1_{A^{c}} H_{2}\right) \leq$ $1_{A} \Pi_{\mathcal{G}}\left(H_{1}\right)+1_{A^{c}} \Pi_{\mathcal{G}}\left(H_{2}\right)$. In particular, $1_{A} \Pi_{\mathcal{G}}\left(1_{A} H_{1}+1_{A^{c}} H_{2}\right) \leq 1_{A} \Pi_{\mathcal{G}}\left(H_{1}\right)$. The other direction follows by setting $\tilde{H}=1_{A} H_{1}+1_{A^{c}} H_{2}$ then as before

$$
1_{A} \Pi_{\mathcal{G}}\left(H_{1}\right)=1_{A} \Pi_{\mathcal{G}}\left(1_{A} \tilde{H}+1_{A^{c}} H_{2}\right) \leq 1_{A} \Pi_{\mathcal{G}}(\tilde{H}) .
$$

Switching the role of $H_{1}$ and $H_{2}$ yields then the desired conclusion.

Other possible axioms which we will consider in a dynamic setting are as follows:

- $\mathcal{G}$-Positive Homogeneity: For $H \in L^{\infty}(\mathcal{F}) \Pi_{\mathcal{G}}(\lambda H)=\lambda \Pi_{\mathcal{G}}(H)$ for all $\lambda \in L_{+}^{\infty}(\mathcal{G})$. 
- Continuity: For any bounded sequence $\left(H_{n}\right)$ which converges a.s. to $H$

$$
\Pi_{\mathcal{G}}(H)=\lim _{n} \Pi_{\mathcal{G}}\left(H_{n}\right)
$$

- $p$-norm boundedness: There exists $p \in(1, \infty), \lambda \in L_{+}^{\infty}(\mathcal{G})$, and a measure $\overline{\mathbb{P}}$ having the same zero sets as $\mathbb{P}$ such that for $H \in L^{\infty}(\mathcal{F})$

$$
\Pi_{\mathcal{G}}(H) \leq \lambda \int\left(|H|+|H|^{p}\right) d \overline{\mathbb{P}}_{\mathcal{G}}
$$

We will also refer to the continuity axiom as 'continuity with respect to a.s. bounded convergence,' if there is any ambiguity. If $\Pi$ is a $\mathcal{G}$-conditional evaluation which is additionally assumed to be positively homogeneous then we call $\Pi$ a $\mathcal{G}$-conditional coherent evaluation. For a further discussion of these axioms see also Artzner et al. (1999). Note that many similar axioms for premium principles can be found in the literature, see for instance Deprez and Gerber (1985) or Kaas et al. (2008). Conditional evaluations in a dynamic setting have been considered for instance in Frittelli and Gianin (2004), Roorda et al. (2005), Ruszczynski and Shapiro (2006), Delbaen (2006), Artzner et al. (2007), Klöppel and Schweizer (2007), Jobert and Rogers (2008), Barrieu and El Karoui (2009), and Cheridito and Kupper (2011). Classical examples of (conditional) evaluations are, see for instance Kaas et al. (2008):

Examples 2.3 - Conditional Mean-Variance principle:

$$
\Pi_{\mathcal{G}}^{v}(H)=\mathbb{E}_{\mathcal{G}}[H]+\frac{1}{2} \alpha \operatorname{Var}_{\mathcal{G}}[H], \quad \alpha \geq 0 .
$$

- Conditional Standard-Deviation principle:

$$
\Pi_{\mathcal{G}}^{s t}(H)=\mathbb{E}_{\mathcal{G}}[H]+\beta \sqrt{\operatorname{Var}_{\mathcal{G}}[H]}, \quad \beta \geq 0 .
$$

- Conditional Semi-Deviation principle:

$$
\Pi_{\mathcal{G}}^{s}(X)=\mathbb{E}_{\mathcal{G}}[H]+\lambda\left|\mathbb{E}_{\mathcal{G}}\left[\left(H-\mathbb{E}_{\mathcal{G}}[H]\right)_{+}^{q}\right]\right|^{1 / q}, \quad \lambda \geq 0, \quad q \in[1, \infty),
$$

where $x_{+}$is 0 if $x<0$ and $x$ else.

- Conditional Average Value at Risk principle:

$$
\Pi_{\mathcal{G}}^{A V @ R}(H)=\mathbb{E}_{\mathcal{G}}[H]+\delta A V @ R_{\mathcal{G}}^{\alpha}\left(H-\mathbb{E}_{\mathcal{G}}[H]\right), \quad \delta \geq 0
$$

where $A V @ R_{\mathcal{G}}^{\alpha}(H)=\frac{1}{\alpha} \int_{0}^{\alpha} V @ R_{\mathcal{G}}^{\lambda}(H) d \lambda, \quad \alpha \in(0,1]$ and $V @ R_{\mathcal{G}}^{\lambda}(H)$ corresponds to computing the Value at Risk of $H$ at the confidence level $\lambda$ with the available information $\mathcal{G}$.

- Conditional Exponential principle:

$$
\Pi_{\mathcal{G}}^{v}(H)=\gamma \log \left(\mathbb{E}_{\mathcal{G}}[\exp \{H / \gamma\}]\right), \quad \gamma>0
$$


Apart from the exponential principle the evaluations above are generally not monotone. However, they are continuous, $\mathcal{G}$-convex, $\mathcal{G}$-cash invariant and satisfy the local property. In particular, they are $\mathcal{G}$-conditional evaluations. The Standard-Deviation principle, the Average Value at Risk principle and the Semi-Deviation principle additionally satisfy $\mathcal{G}$-positive homogeneity while the Mean-Variance principle is $p$-norm bounded (with $\overline{\mathbb{P}}=\mathbb{P}$ ). The Average Value at Risk principle and the Semi-Deviation principle are monotone if $\lambda$ or $\delta$ are in $[0,1]$.

We will need some duality results. For a $\sigma$-algebra $\mathcal{G} \subset \mathcal{F}$, denote $\mathcal{Q}_{\mathcal{G}}=\left\{\xi \in L^{1}(\mathcal{F}) \mid \mathbb{E}_{\mathcal{G}}[\xi]=\right.$ $1\}$ and $\mathcal{Q}_{\mathcal{G}}^{+}:=\left\{\xi \in L_{+}^{1}(\mathcal{F}) \mid \mathbb{E}_{\mathcal{G}}[\xi]=1\right\}$. In other words, given the information $\mathcal{G}, \mathcal{Q}_{\mathcal{G}}$ is the set of all signed measures and $\mathcal{Q}_{\mathcal{G}}^{+}$is the set of all probability measures. Therefore, conditional on our starting information $\mathcal{G}$, we may identify every $\xi \in \mathcal{Q}_{\mathcal{G}}$ with a signed measure, and every $\xi \in \mathcal{Q}_{\mathcal{G}}^{+}$with a probability measure. For instance, for $\xi \in \mathcal{Q}_{\mathcal{G}}^{+}$we can define the corresponding conditional probability measure $\mathbb{Q}_{\mathcal{G}}^{\xi}(A):=\mathbb{E}_{\mathcal{G}}\left[\xi I_{A}\right]$, and its conditional density as $\frac{d \mathbb{Q}_{\mathcal{G}}^{\xi}}{d \mathbb{P}_{\mathcal{G}}}:=\xi$.

Recall that by standard duality results we have that $\Pi_{\mathcal{G}}$ is coherent if and only if

$$
\Pi_{\mathcal{G}}(H)=\operatorname{ess}_{\sup _{\xi \in M}} \mathbb{E}_{\mathcal{G}}[\xi H]
$$

for a unique closed, convex set $M \subset \mathcal{Q}_{\mathcal{G}}$. For the precise definition of the essential supremum, see the Appendix. $\xi$ is often interpreted as a weighting function for the different scenarios $\omega$, or as a test or stress measure. By taking the supremum, a worst-case approach is being taken. For instance in the good-deal bound literature mentioned in the introduction the supremum is taken over all pricing kernels with a density admitting a variance smaller than a certain constant.

Generally it holds for all conditional evaluations that for $\Pi_{\mathcal{G}}^{*}$ defined by

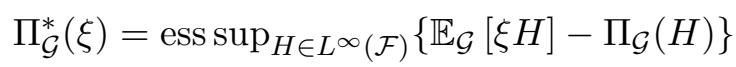

we have that

$$
\Pi_{\mathcal{G}}(H)=\operatorname{ess}_{\sup } \operatorname{se}_{\mathcal{G}}\left\{\mathbb{E}_{\mathcal{G}}[\xi H]-\Pi_{\mathcal{G}}^{*}(\xi)\right\},
$$

see for instance Delbaen (2006) or Cheridito and Kupper (2011). $\Pi_{\mathcal{G}}^{*}$ is also called the penalty function corresponding to $\Pi_{\mathcal{G}}$, and $-\Pi_{\mathcal{G}}^{*}(\xi)$ may be seen as the plausibility of the density $\xi$. The dual representations (2.1) and (2.2) are often interpreted as robust expectations with respect to different priors, see Hansen and Sargent (2001), or Hansen and Sargent (2007).

Of course our results also hold without conditioning on $\mathcal{G}$. In this case $\mathcal{G}$ is chosen to be the trivial $\sigma$-algebra. However, for our dynamic analysis in the later sections it will simplify matters if we do our analysis conditional on some information available to the agent.

\section{Market-Consistent Pricing}

\subsection{Market-consistency and two step market evaluations}

Let $(\Omega, \mathcal{F}, \mathbb{P})$ be the underlying probability space. Let $\mathcal{G} \subset \mathcal{F}$ be a $\sigma$-algebra whose information is initially available to the agent. Let $S=\left(S^{1}, \ldots, S^{n}\right)$ be the $n$-dimensional price process of $n$ traded stocks and denote by $\overline{\mathcal{F}}^{S} \subset \mathcal{F}$ the $\sigma$-algebra generated by $S$. Furthermore, we denote by $\mathcal{F}^{S}$ the $\sigma$-algebra given by the stock process and our starting information $\mathcal{G}$, i.e., $\mathcal{F}^{S}:=\overline{\mathcal{F}}^{S} \vee \mathcal{G}:=\sigma\left(\overline{\mathcal{F}}^{S}, \mathcal{G}\right)$. The financial market given by the $n$-dimensional stock process $S$ should be arbitrage free and complete, i.e., all derivatives which conditional on $\mathcal{G}$ only depend 
on $S$ are perfectly hedgeable and there exists a unique probability measure $\mathbb{Q}_{\mathcal{G}} \in \mathcal{Q}_{\mathcal{G}}^{+}$such that $S$ is a martingale under $\mathbb{Q}_{\mathcal{G}}$ (componentwise). Furthermore, $\mathbb{Q}_{\mathcal{G}}$ is assumed to be a.s. equivalent to $\mathbb{P}_{\mathcal{G}}$, in the sense that its conditional density is positive.

Since $\mathcal{F}^{S}$ is in general a strict subset of $\mathcal{F}$, the market given by all $\mathcal{F}$-measurable payoffs is incomplete. For instance, we could have an untraded insurance process which is correlated with the traded assets $S$ but not perfectly replicable.

In the financial market the martingale measure $\mathbb{Q}_{\mathcal{G}}$ defines the (linear) no-arbitrage pricing operator $\Pi_{\mathcal{G}}^{f}: L^{\infty}\left(\mathcal{F}^{S}\right) \rightarrow L^{\infty}(\mathcal{G})$ given by

$$
\Pi_{\mathcal{G}}^{f}\left(H^{S}\right):=\mathbb{E}^{\mathbb{Q}_{\mathcal{G}}}\left[H^{S}\right]:=\int_{\Omega} H^{S}(\omega) \mathbb{Q}_{\mathcal{G}}(d \omega)=\mathbb{E}_{\mathcal{G}}\left[\xi^{\mathbb{Q}_{\mathcal{G}}} H\right]
$$

where $\xi^{\mathbb{Q}_{\mathcal{G}}}$ is the density in $\mathcal{Q}_{\mathcal{G}}^{+}$with which $\mathbb{Q}_{\mathcal{G}}$ may be identified. Note that only the martingale measure on $\mathcal{F}^{S}$ is unique while on the filtration $\mathcal{F}$ there can be infinitely many martingale measures.

The next definition extends the notion of cash invariance to all assets traded in the financial market. For identical or similar notions of market-consistency see also Cont (2006), Kupper et al. (2008), Malamud et al. (2008), or Artzner and Eisele (2010).

Definition 3.1 An evaluation is called market-consistent if for any financial payoff $H^{S} \in$ $L^{\infty}\left(\mathcal{F}^{S}\right)$ and $H \in L^{\infty}(\mathcal{F})$

$$
\Pi_{\mathcal{G}}\left(H^{S}+H\right)=\mathbb{E}^{\mathbb{Q}_{\mathcal{G}}}\left[H^{S}\right]+\Pi_{\mathcal{G}}(H) .
$$

Market-consistency postulates that liquidly traded assets and payoffs replicable by them should not carry any risk as they can be converted to cash at any time. It follows immediately from the definition that a market-consistent evaluation cannot be 'improved' by hedging.

Remark 3.2 Our definition of market-consistency requires that we have liquidly traded assets constituting a complete financial market. If the financial market is not complete then there are two possibilities which may still validate our approach:

(1.) One could use certain financial derivatives as additional hedging instruments to make the financial market complete. For results in this direction, see Jacod and Protter (2010) and the references therein. Note that in our setting $S$ has to be finite-dimensional. Therefore, only finitely many additional hedging instruments are allowed. However, many stochastic volatility models like the Heston model can be completed in this way.

(2.) One could remove certain financial assets as possible hedging instruments. In some cases the remaining assets might constitute a complete financial market.

The next proposition shows that market-consistency is already implied by the assumption that purely hedgeable derivatives should be valued according to the amount of capital necessary to replicate them. Furthermore, it shows that, in case that $\Pi_{\mathcal{G}}$ is monotone, market-consistency is equivalent with the no-arbitrage principle in the entire market.

Proposition 3.3 For a conditional evaluation $\Pi_{\mathcal{G}}: L^{\infty}(\mathcal{F}) \rightarrow L^{\infty}(\mathcal{G})$ the following statements are equivalent:

(i) $\Pi_{\mathcal{G}}\left(H^{S}\right)=\mathbb{E}^{\mathbb{Q}_{\mathcal{G}}}\left[H^{S}\right]$ for any financial payoff $H^{S} \in L^{\infty}\left(\mathcal{F}^{S}\right)$. 
(ii) There exists a penalty function ${ }^{*} c_{\mathcal{G}}: \mathcal{Q}_{\mathcal{F}^{S}}=\left\{Z \in L^{1}(\mathcal{F}) \mid \mathbb{E}_{\mathcal{F}^{S}}[Z]=1\right\} \rightarrow \mathbb{R} \cup\{\infty\}$ such that we have for every $H \in L^{\infty}(\mathcal{F})$

$$
\Pi_{\mathcal{G}}(H)=\operatorname{ess}_{\sup _{Z \in \mathcal{Q}_{\mathcal{F}}}}\left\{\mathbb{E}^{\mathbb{Q}_{\mathcal{G}}}[Z H]-c_{\mathcal{G}}(Z)\right\} .
$$

(iii) $\Pi_{\mathcal{G}}$ is market-consistent.

Furthermore, in the case that $\Pi_{\mathcal{G}}$ is additionally assumed to be monotone, market-consistency is equivalent with any payoff being evaluated between its super-and sub-replication price.

In particular, in the case of monotonicity, we could have also defined market-consistency by stating that the evaluation of every payoff should respect the no-arbitrage principle.

Examples of market-consistent evaluations arise if an agent starts with a (usually non market-consistent) evaluation $\Pi_{\mathcal{G}}$ and then tries to reduce his risk by hedging. Specifically denote by $\mathcal{A}$ all admissible hedging strategies $\pi$ (defined in an appropriate way), by $\mathcal{M}_{\mathcal{G}} \subset$ $\mathcal{Q}_{\mathcal{G}}^{+}:=\mathcal{Q}_{\mathcal{G}} \cap L_{+}^{1}(\mathcal{F})$ the set of all local martingale measures, by $\tilde{S}$ the discounted stock process, and by $\Pi_{\mathcal{G}}^{*}$ the penalty function corresponding to $\Pi_{\mathcal{G}}$. It can be shown, see for instance Barrieu and El Karoui (2009), or Toussaint and Sircar (2011), that under appropriate assumptions

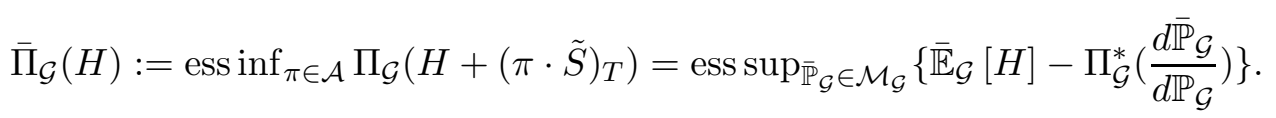

Note that by definition $\bar{\Pi}_{\mathcal{G}}$ is market-consistent. Therefore, one way of obtaining marketconsistent evaluations is to intersect the test measures in the dual representations (2.1) and (2.2) above with local martingale measures. This is a class of examples which arises naturally when starting with an evaluation $\Pi_{\mathcal{G}}$. However, the new evaluation $\bar{\Pi}_{\mathcal{G}}$ may be hard to compute, and the structure and interpretation of the original evaluation $\Pi_{\mathcal{G}}$ is lost. For instance, if

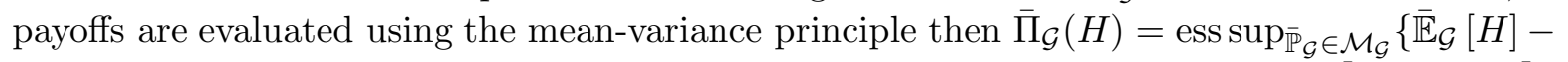
$\left.\frac{1}{2 \alpha} C_{\mathcal{G}}\left(\overline{\mathbb{P}}_{\mathcal{G}} \mid \mathbb{P}_{\mathcal{G}}\right)\right\}$, where $C_{\mathcal{G}}$ is the relative Gini index defined by $C_{\mathcal{G}}\left(\overline{\mathbb{P}}_{\mathcal{G}} \mid \mathbb{P}_{\mathcal{G}}\right)=\mathbb{E}_{\mathcal{G}}\left[\left(\frac{d \overline{\mathbb{P}}_{\mathcal{G}}}{d \mathbb{P}_{\mathcal{G}}}\right)^{2}-1\right]$. For an overview about mean-variance hedging, see Schweizer (2010) and the reference therein. Now note that two important reasons for the popularity of the Mean-variance principle are: (a) it has a straight-forward interpretation; (b) it is easy to compute. However, while the new evaluation, $\bar{\Pi}_{\mathcal{G}}(H)$ is market-consistent (since it uses risk adjusted probabilities), it is neither easy to compute nor does it directly relate to the variance of the payoff $H$ from which we started.

Consequently, in this paper we propose a new class of market-consistent evaluations which we will call two step market evaluations. Extending standard actuarial principles with two step market evaluations will have the advantage that the extensions can be computed easily and that the interpretation of our starting principles can be preserved. Furthermore, we will show that two step market evaluations in general have many other appealing properties. A strong argument for the use of two step market evaluations will be provided in the Chapter 4. There we show that any insurance company which wants to apply a market-consistent and time-consistent evaluation, has to use a two step market evaluation, in a setting where the stock process is continuous and the insurance process is only revealed at fixed time instances (or more generally at predictable stopping times).

${ }^{*}$ A function $c$ is called a penalty function if it is convex, lower-semicontinuous and ess inf $c=0$. 
We will start with evaluations like the one from our Examples 2.3. Then we will give the corresponding market-consistent evaluations which do not arise from hedging but from operator splitting. Namely, in a first step we compute the value of the position $H$ by replacing the measure $\mathbb{P} \mid \mathcal{G}$ with the measure $\mathbb{P} \mid \mathcal{F}^{S}$, i.e., we compute Mean-Variance principle, the StandardDeviation principle, etc., of $H$, conditional on $\mathcal{G}$ and the values of the stocks $S$. Then for every different future value of the stock price we get a different evaluation. However, since payoff depending only on the stock prices are perfectly hedgeable one could argue that these remaining evaluations do not contain any risk. Therefore, the total value of the position $H$ should be equal to the initial capital needed to hedge the different evaluations, obtained in the first step, which depend on $S$. This corresponds to taking in a second step the expectation with respect to the risk adjusted probability measure $\mathbb{Q}_{\mathcal{G}}$ coming from the financial market. This procedure is computationally tractable and preserves the evaluation principles considered in the beginning. These principles are applied to the risk which remains after having conditioned on $S$. We then get the following market-consistent examples:

Examples 3.4 - Two step Mean-Variance principle:

$$
\Pi_{\mathcal{G}}^{v}(H)=\mathbb{E}^{\mathbb{Q}_{\mathcal{G}}}\left[\mathbb{E}_{\mathcal{F}^{S}}[H]+\frac{1}{2} \alpha \operatorname{Var}_{\mathcal{F} S}[H]\right], \quad \alpha \geq 0 .
$$

- Two step Standard-Deviation principle:

$$
\Pi_{\mathcal{G}}^{s}(H)=\mathbb{E}^{\mathbb{Q}_{\mathcal{G}}}\left[\mathbb{E}_{\mathcal{F}^{S}}[H]+\beta \sqrt{\operatorname{Var}_{\mathcal{F}^{S}}[H]}\right], \quad \beta \geq 0 .
$$

- Two step Semi-Deviation principle:

$$
\Pi_{\mathcal{G}}^{s}(X)=\mathbb{E}^{\mathbb{Q}_{\mathcal{G}}}\left[\mathbb{E}_{\mathcal{F}^{S}}[H]+\lambda\left|\mathbb{E}_{\mathcal{F}^{S}}\left[\left(H-\mathbb{E}_{\mathcal{F}^{S}}[H]\right)_{+}^{q}\right]\right|^{1 / q}\right], \quad \lambda \geq 0, \quad q \in[1, \infty) .
$$

- Two step Average Value at Risk principle:

$$
\Pi_{\mathcal{G}}^{A V @ R}(H)=\mathbb{E}^{\mathbb{Q}_{\mathcal{G}}}\left[\mathbb{E}_{\mathcal{F}^{S}}[H]+\delta A V @ R_{\mathcal{F} S}^{\alpha}\left(H-\mathbb{E}_{\mathcal{F}^{S}}[H]\right)\right], \quad \delta \geq 0 .
$$

- Two step Exponential principle:

$$
\Pi_{\mathcal{G}}(H)=\mathbb{E}^{\mathbb{Q}_{\mathcal{G}}}\left[\gamma \log \left(\mathbb{E}_{\mathcal{F}^{S}}[\exp \{H / \gamma\}]\right)\right], \quad \gamma>0
$$

A standard deviation principle, which is different than the one above but is also obtained by first conditioning on the future stock price, is considered in Møller (2002). The last example is known in the literature as the indifference price of $H$ under an exponential utility function. It arises in an incomplete market when an agent maximizes his exponential utility through dynamic trading, see for instance Musiela and Zariphopoulou (2004b). The indifference price for a claim $H$ is then defined as the amount of cash the agent is willing to pay for the right to receive $H$ such that he is no worse off in expected utility terms than he would have been without the claim. For references on indifference pricing see the introduction. The examples above motivate the following definition: 
Definition 3.5 We call a $\Pi_{\mathcal{G}}: L^{\infty}(\mathcal{F}) \rightarrow L^{\infty}(\mathcal{G})$ a two step market evaluation if there exists an $\mathcal{F}^{S}$-conditional valuation $\Pi_{\mathcal{F}^{S}}: L^{\infty}(\mathcal{F}) \rightarrow L^{\infty}\left(\mathcal{F}^{S}\right)$ such that

$$
\Pi_{\mathcal{G}}(H)=\mathbb{E}^{\mathbb{Q}_{\mathcal{G}}}\left[\Pi_{\mathcal{F} S}(H)\right] .
$$

Note that in case that there is no financial market, i.e., $S=0$, our two step evaluations reduce to the standard actuarial principles. On the other hand if $\mathcal{F}^{S}=\mathcal{F}$, i.e., if the financial market gives the entire filtration, of course $\Pi_{\mathcal{G}}(H)=\mathbb{E}^{\mathbb{Q}_{\mathcal{G}}}[H]$.

Example 3.6 Another example for a two step evaluation defined above arises when combining hedging with an Average Value at Risk principle. Specifically set

$$
\Pi_{\mathcal{G}}(H):=\operatorname{essinf}_{\pi \in \mathcal{A}} \Pi_{\mathcal{G}}^{A V @ R}\left(H+(\pi \cdot \tilde{S})_{T}\right) .
$$

Define

$$
M:=\left\{\overline{\mathbb{Q}}_{\mathcal{G}} \in \mathcal{M}_{\mathcal{G}} \mid 1-\delta \leq \frac{d \overline{\mathbb{Q}}_{\mathcal{G}}}{d \mathbb{P}_{\mathcal{G}}} \leq 1+\delta \frac{1-\alpha}{\alpha}\right\}
$$

where $\delta>0$ and the risk level $\alpha \in(0,1]$ have been introduced in the Examples 2.3. (Note that if $\delta=0$ then $\Pi_{\mathcal{G}}^{A V @ R}(H)=\mathbb{E}_{\mathcal{G}}[H]$.)

From (3.1) and the dual representation of the Average-Value at Risk principle (see for instance Föllmer and Schied (2004)) it follows that

$$
\Pi_{\mathcal{G}}(H)=\operatorname{ess}_{\sup _{\overline{\mathbb{Q}}_{\mathcal{G}} \in M}} \mathbb{E}^{\overline{\mathbb{Q}}_{\mathcal{G}}}[H]
$$

Let

$$
M^{\prime}:=\left\{Z \in \mathcal{Q}_{\mathcal{F} S}^{+} \mid(1-\delta) \frac{d \mathbb{P}_{\mathcal{G}}}{d \mathbb{Q}_{\mathcal{G}}} \leq Z \leq \frac{d \mathbb{P}_{\mathcal{G}}}{d \mathbb{Q}_{\mathcal{G}}}\left(1+\delta \frac{1-\alpha}{\alpha}\right)\right\}
$$

Note that $\frac{d \mathbb{Q}_{\mathcal{G}}}{d \mathbb{P}_{\mathcal{G}}} M^{\prime}=M$. Furthermore, $M^{\prime}$ satisfies the concatenation property on $\mathcal{F}^{S}$, also called rectangular property or $m$-stability ${ }^{\dagger}$. It may be seen that as a result

$$
\Pi_{\mathcal{F} S}(H):=\operatorname{ess}_{\sup _{Z \in M^{\prime}}} \mathbb{E}_{\mathcal{F} S}[Z H]
$$

is an $\mathcal{F}^{S}$-conditional evaluation and it holds that

$$
\Pi_{\mathcal{G}}(H)=\mathbb{E}^{\mathbb{Q}_{\mathcal{G}}}\left[\Pi_{\mathcal{F} S}(H)\right],
$$

compare also with Theorem 3.10 below. In particular, combining hedging with the AverageValue at Risk principle gives an example of the two step procedure we explained above.

Example 3.7 Our last example is given by the super-replication price of a contingent claim. The super-replication price is given by

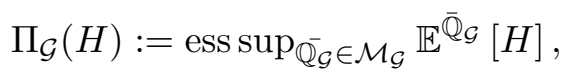

It is straight-forward to check that $\mathcal{M}_{\mathcal{G}}=\frac{d \mathbb{Q}_{\mathcal{G}}}{d \mathbb{P}_{\mathcal{G}}} \mathcal{Q}_{\mathcal{F} S}^{+}$. Clearly,

$$
\Pi_{\mathcal{F} S}(H):=\operatorname{ess}_{\sup _{Z \in \mathcal{Q}_{\mathcal{F} S}^{+}}} \mathbb{E}_{\mathcal{F} S}[Z H]
$$

\footnotetext{
${ }^{\dagger}$ The rectangular property or $m$-stability on a $\sigma$-algebra $\overline{\mathcal{G}}$ postulates that for every $A \in \mathcal{F}^{S}$ and $Z_{1}, Z_{2} \in M^{\prime}$ we have that $I_{A} Z_{1}+I_{A^{c}} Z_{2} \in M^{\prime}$, see for instance Chen and Epstein (2002) or Delbaen (2006).
} 
is an $\mathcal{F}^{S}$-conditional evaluation. It computes the essential supremum conditional on $\mathcal{F}^{S}$. Since $\mathcal{Q}_{\mathcal{F} S}^{+}$is $m$-stable it can be seen that

$$
\Pi_{\mathcal{G}}(H)=\mathbb{E}^{\mathbb{Q}}{ }_{\mathcal{G}}\left[\Pi_{\mathcal{F}^{S}}(H)\right]
$$

In particular, the super-replication price is a two step evaluation.

Remark 3.8 Note that equity linked insurance payoffs are typically of the form $H=Y_{T}^{(n)} f\left(S_{T}\right)$ where $f\left(S_{T}\right)$ is a financial derivative and $Y_{T}^{(n)}$ are the number of policy holder who survived up to time $T$ (out of an initial cohort of $n$ ), see for instance Møller (2002). In the special case that the financial and the mortality risk are independent a two step market evaluation would yield

$$
\Pi_{\mathcal{G}}(H)=\mathbb{E}^{\mathbb{Q}_{\mathcal{G}}}\left[\Pi_{\mathcal{F}^{S}}\left(f\left(S_{T}\right) Y_{T}^{(n)}\right)\right]=\mathbb{E}^{\mathbb{Q}_{\mathcal{G}}}\left[f\left(S_{T}\right)\right] \Pi_{\mathcal{G}}\left(Y_{T}^{(n)}\right) .
$$

Note that the structure obtained in this special case is similar to the market-consistent valuation method suggested in Chapter 2.6 in Wüthrich et al. (2008).

Two step market valuations provide a rich class of market-consistent evaluations with a clear underlying intuition. They appear as indifference-price with an exponential utility, in hedging with an Average Value at Risk principle, and in the super-replication price of an contingent claim. Two step market valuations are also useful in optimization problems since the maximum may be computed by a two step procedure using Bellman's principle: first one can compute a value function conditioned on the stock process, and then in a second step one can compute the optimum by maximizing the value function obtained in Step 1 under the pricing measure.

Note that contrary to other evaluations two step market evaluations can be directly converted into an equivalent evaluation which takes the stock process as the numeraire. That is, assume payoffs $\tilde{H}$ are expressed in units of the $i$-th stock, $S^{i}$ for $i \in\{1, \ldots, n\}$, i.e., $\tilde{H}=H / S_{T}^{i}$. An agent who wants to use the evaluation $\Pi_{\mathcal{G}}$ but wants to express everything in units of $S^{i}$ obviously should use the evaluation $\tilde{\Pi}_{\mathcal{G}}$ which satisfies $S_{0}^{i} \tilde{\Pi}_{\mathcal{G}}(\tilde{H})=\Pi_{\mathcal{G}}(H)$. The evaluation $\tilde{\Pi}_{\mathcal{G}}$ often might not be easy to characterize directly, for instance with a dual representation and a penalty function. However, a two step market evaluation remains a two step market evaluation under the change of numeraire. Moreover, one can just define the new penalty function of $\tilde{\Pi}_{\mathcal{F} S}$ as the penalty function of $\Pi_{\mathcal{F}^{S}}$ in units of $S_{T}^{i}$. That is we set

$$
\tilde{\Pi}_{\mathcal{F} S}^{*}(\xi):=\frac{\Pi_{\mathcal{F} S}^{*}(\xi)}{S_{T}^{i}} \text { and } \tilde{\Pi}_{\mathcal{F} S}(\tilde{H}):=\operatorname{ess}_{\sup _{\xi \in L^{1}(\mathcal{F})}}\left\{\mathbb{E}_{\mathcal{F} S}[\tilde{H}]-\tilde{\Pi}_{\mathcal{F} S}^{*}(\xi)\right\} .
$$

Denote by $\tilde{\mathbb{Q}}_{\mathcal{G}}$ the unique equivalent martingale measure on $\mathcal{F}^{S}$ with numeraire $S^{i}$ that is

$$
\frac{d \tilde{\mathbb{Q}}_{\mathcal{G}}}{d \mathbb{P}_{\mathcal{G}}}:=\frac{S_{T}^{i}}{S_{0}^{i}} \frac{d \mathbb{Q}_{\mathcal{G}}}{d \mathbb{P}_{\mathcal{G}}}
$$

Then we obtain

$$
\tilde{\Pi}_{\mathcal{G}}(\tilde{H})=\frac{\Pi_{\mathcal{G}}(H)}{S_{0}^{i}}=\mathbb{E}^{\mathbb{Q}_{\mathcal{G}}}\left[\frac{S_{T}^{i}}{S_{0}^{i}} \operatorname{ess}^{\sup _{\xi \in L^{1}}(\mathcal{F})}\left\{\mathbb{E}_{\mathcal{F}^{S}}[\tilde{H}]-\tilde{\Pi}_{\mathcal{F} S}^{*}(\xi)\right\}\right]=\mathbb{E}^{\tilde{\mathbb{Q}}_{\mathcal{G}}}\left[\tilde{\Pi}_{\mathcal{F}^{S}}(\tilde{H})\right] .
$$

We will summarize the last paragraph in the following proposition: 
Proposition 3.9 If $\Pi_{\mathcal{G}}$ is a two step market evaluation then $\tilde{\Pi}_{\mathcal{G}}$ is a two step market evaluation as well. Furthermore, the penalty function of $\tilde{\Pi}_{\mathcal{F} S}$ is given by the penalty function of $\Pi_{\mathcal{F} S}$ converted into units of stock $i$.

Note that for evaluations which are not two step an easy conversion of the penalty function as given in the last proposition usually only works if the numeraire is deterministic.

It is not hard to see that a two step market valuation is always market-consistent, see the theorems below. On the other hand our Example 3.12 below shows that a market-consistent valuation is not necessarily a two step market valuation.

\subsection{An axiomatic characterization of two step market evaluations}

Notice that the Examples 3.4 all satisfy the market local property, i.e.:

- For every $A \in \mathcal{F}^{S}$ and $H \in L_{+}^{\infty}(\mathcal{F})$

$$
\Pi_{\mathcal{G}}(H)=\Pi_{\mathcal{G}}\left(I_{A} H\right)+\Pi_{\mathcal{G}}\left(I_{A^{c}} H\right) .
$$

The binary options $I_{A} H$ and $I_{A^{c}} H$ can be seen as insurances against the events $A$ or $A^{c}$ respectively. For example if $A$ happens then the owner of the option $I_{A} H$ gets a non-negative amount $H$ which possibly depends on the insurance process. If the evaluation $\Pi_{\mathcal{G}}$ is assumed to be sublinear, the value of the insurance contract $I_{A} H$ plus the value of the insurance contract $I_{A^{c}} H$ should be larger than the value of $H=I_{A} H+I_{A^{c}} H$. The economic reason is that if the valuation of $H$ is decomposed into the sum of the evaluations of the binary insurance contracts then the additional uncertainty given by the event $A$ for each binary contract, should lead to an increase of the total insurance premium. The local property however postulates that the uncertainty added to the payoff $H$ by an event from the financial market should not carry any extra premium. We will see in Section 4 that, in a setting with a correlated stock and insurance process, the local property is satisfied for a time-consistent and market-consistent evaluation if the stock process in continuous and the value of the insurance process for is revealed at fixed time instances (or more generally at predictable stopping times).

Now the question in which we are interested in the remainder of this chapter, is the following: Given a conditional evaluation $\Pi_{\mathcal{G}}$ does then its market-consistency and the market local property imply that every position $H$ has to be priced with a two step market valuation? Our results below will actually show that this statement holds.

The following theorem shows that market-consistency and the market local property in the coherent case are equivalent to two step market evaluations. Furthermore, it gives an explicit formula for $\Pi_{\mathcal{F} S}$.

Theorem 3.10 The following statements are equivalent:

(i) $\Pi_{\mathcal{G}}$ is a coherent market-consistent $\mathcal{G}$-conditional evaluation which satisfies the market local property.

(ii) There exists an $\mathcal{F}^{S}$-conditional coherent evaluation $\Pi_{\mathcal{F}^{S}}: L^{\infty}(\mathcal{F}) \rightarrow L^{\infty}\left(\mathcal{F}^{S}\right)$ such that $\Pi_{\mathcal{G}}(H)=\mathbb{E}^{\mathbb{Q}_{\mathcal{G}}}\left[\Pi_{\mathcal{F}^{S}}(H)\right]$. Furthermore, $\Pi_{\mathcal{F}^{S}}(H)=\operatorname{ess}_{\sup _{Z \in M^{\prime}}} \mathbb{E}_{\mathcal{F}^{S}}[Z H]$, with

$$
M^{\prime}:=\left(\frac{d \mathbb{Q}_{\mathcal{G}}}{d \mathbb{P}_{\mathcal{G}}}\right)^{-1} M \subset\left\{Z \in L^{1}(\mathcal{F}) \mid \mathbb{E}_{\mathcal{F}^{S}}[Z]=1\right\}=\mathcal{Q}_{\mathcal{F}^{S}},
$$


and $M$ given by (2.1).

The assumptions of the theorem are satisfied for all our examples above except for the MeanVariance and the Exponential market-consistent principles. The reason is that these do not satisfy positive homogeneity. However, in the case that the filtration $\mathcal{F}^{S}$ is generated by countably many sets, and $\Pi_{\mathcal{G}}$ is continuous, and monotone or $p$-norm bounded, we can prove that an evaluation has to be a two step market evaluation without the assumption of positive homogeneity.

Theorem 3.11 Suppose that $\mathcal{F}^{S}$ is generated by countably many sets. Then the following statements are equivalent:

(i) $\Pi_{\mathcal{G}}$ is a monotone, continuous market-consistent $\mathcal{G}$-conditional evaluation which satisfies the market local property.

(ii) There exists a monotone, continuous $\mathcal{F}^{S}$-conditional evaluation $\Pi_{\mathcal{F}^{S}}: L^{\infty}(\mathcal{F}) \rightarrow L^{\infty}\left(\mathcal{F}^{S}\right)$ such that

$$
\Pi_{\mathcal{G}}(H)=\mathbb{E}^{\mathbb{Q}_{\mathcal{G}}}\left[\Pi_{\mathcal{F} S}(H)\right] .
$$

Furthermore, if $\Pi_{\mathcal{G}}$ is additionally assumed to be p-norm bounded then the statement also holds without the monotonicity assumption in (i) and (ii).

The Mean-Variance principle is not monotone but it is $p$-norm bounded. In particular, Theorems 3.10-3.11 include all our examples. Theorem 3.11 further applies to the Exponential principle, and the Average Value at Risk and the Semi-Deviation principle if $\lambda$ and $\delta$ only take values between 0 and 1 .

The proof of the last theorem heavily relies on the assumed continuity of our evaluations which was not needed in Theorem 3.10. The evaluation $\Pi_{\mathcal{F}^{S}}$ will be obtained only as an a.s. limit using the martingale convergence theorem without an explicit formula.

Note that the market-consistency of an evaluation $\Pi$ does not necessarily imply that we get a representation as in Theorem 3.10 and Theorem 3.11, since there are market-consistent evaluations not satisfying the market local property. This can be seen from the following example:

Example 3.12 Assume that $\mathcal{G}$ is trivial and let $Z_{1}$ and $Z_{2}$ be densities independent of $S$ with $Z_{1} \neq Z_{2}$. Then $Z_{i}$ are also independent of $\frac{d \mathbb{Q}}{d \mathbb{P}}$. Now suppose that the agent is not sure if he should trust the density $\frac{d \mathbb{Q}}{d \mathbb{P}} Z_{1}$ or $\frac{d \mathbb{Q}}{d \mathbb{P}} Z_{2}$. Therefore, he decides to take a worst-case approach over all convex combinations of $\frac{d \mathbb{Q}}{d \mathbb{P}} Z_{1}$ or $\frac{d \mathbb{Q}}{d \mathbb{P}} Z_{2}$. That is

$$
\Pi(H):=\max _{\overline{\mathbb{P}} \in M} \overline{\mathbb{E}}[H]=\max _{i=1,2} \mathbb{E}\left[\frac{d \mathbb{Q}}{d \mathbb{P}} Z_{i} H\right],
$$

with $M=\left\{\lambda \frac{d \mathbb{Q}}{d \mathbb{P}} Z_{1}+(1-\lambda) \frac{d \mathbb{Q}}{d \mathbb{P}} Z_{2} \mid \lambda \in[0,1]\right\}$. It is straight-forward to check using the independence of $Z_{i}$ and $S$ that $\Pi$ is a market-consistent, coherent evaluation. Let $H \geq 0$ be $\mathcal{F}$ - but not $\mathcal{F}^{S}$-measurable. Assume without loss of generality that the maximum in (3.5) is attained in $i=1$. Now choose a set $A \in \mathcal{F}^{S}$ such that

$$
\mathbb{E}\left[\frac{d \mathbb{Q}}{d \mathbb{P}} Z_{2} I_{A} H\right]>\mathbb{E}\left[\frac{d \mathbb{Q}}{d \mathbb{P}} Z_{1} I_{A} H\right]
$$


Since the maximum in (3.5) is attained in $i=1$ we must have that

$$
\mathbb{E}\left[\frac{d \mathbb{Q}}{d \mathbb{P}} Z_{2} I_{A^{c}} H\right]<\mathbb{E}\left[\frac{d \mathbb{Q}}{d \mathbb{P}} Z_{1} I_{A^{c}} H\right] .
$$

But then we get

$$
\begin{aligned}
\Pi\left(H I_{A}\right)+\Pi\left(H I_{A^{c}}\right) & =\mathbb{E}\left[\frac{d \mathbb{Q}}{d \mathbb{P}} Z_{2} I_{A} H\right]+\mathbb{E}\left[\frac{d \mathbb{Q}}{d \mathbb{P}} Z_{1} I_{A^{c}} H\right] \\
& >\mathbb{E}\left[\frac{d \mathbb{Q}}{d \mathbb{P}} Z_{1} I_{A} H\right]+\mathbb{E}\left[\frac{d \mathbb{Q}}{d \mathbb{P}} Z_{1} I_{A^{c}} H\right]=\Pi(H) .
\end{aligned}
$$

One possible choice for $A$ and $H$ would be to define $H=I_{A} I_{\left\{Z_{2}>Z_{1}\right\}}+I_{A^{c}} I_{\left\{Z_{1}>Z_{2}\right\}}$. Then (3.6) always holds if $A$ is chosen to be a non-zero set with $A \subset\left\{Z_{2}>Z_{1}\right\}$. Furthermore, one can ensure that $\Pi(H)=\mathbb{E}\left[Z_{1} H\right]$ by choosing $A$ such that $\mathbb{Q}\left(A^{c}\right)$ is sufficiently close to one. In particular, (3.7) holds and therefore $\Pi$ defined in (3.5) does not satisfy the market local property. By the direction (ii) $\Rightarrow(\mathrm{i})$ of Theorem 3.10 this implies that $\Pi$ is not a two step market evaluation.

\section{Dynamic Evaluations in continuous time: Time-consistency and Market-consistency}

In this section we will give sufficient conditions in which the market local property, holds in a dynamic setting. Specifically we will obtain that in certain dynamic settings time-consistency and market-consistency imply that all evaluations have to be two step market evaluations. Time-consistency in a dynamic setting often has strong implications. For instance, for general preferences the indifference price of an agent with time-consistent dynamic preferences are recursive if and only if the preferences are cash-invariant, see Theorem 3.4, Cheridito and Kupper (2009).

Subsequently, we fix a finite time horizon $T>0$. Throughout the rest of the paper we assume that additional to the stocks $\left(S_{t}\right)_{0 \leq t \leq T}=\left(\left(S_{t}^{1}, \ldots, S_{t}^{n}\right)\right)_{0 \leq t \leq T}$, we have an untraded insurance process $\left(Y_{t}\right)_{0 \leq t \leq T}$. For the sake of simplicity let us assume that the insurance process is one-dimensional. (The generalization is straight-forward.) Let $\overline{\mathcal{F}}^{S}$ be filtration generated by $S$, and let $\overline{\mathcal{F}}^{Y}$ be the filtration generated by $Y$. We again assume that the financial market is complete while the entire market is incomplete. Denote by $\mathbb{Q}$ the unique martingale measure on $\overline{\mathcal{F}}_{T}^{S}$ with density $\frac{d \mathbb{Q}}{d \mathbb{P}}$.

Define the total information which is available as $\mathcal{F}:=\overline{\mathcal{F}}^{S} \vee \overline{\mathcal{F}}^{Y}:=\sigma\left(\overline{\mathcal{F}}^{S} \cup \overline{\mathcal{F}}^{Y}\right)$. Setting $\mathbb{Q}(A):=\mathbb{E}\left[\frac{d \mathbb{Q}}{d \mathbb{P}} I_{A}\right]$ for $A \in \mathcal{F}_{T}$, we can extend $\mathbb{Q}$ canonically to the whole filtration. We call a collection of mappings $\left(\Pi_{\sigma}\right)_{0 \leq \sigma \leq T}$, a continuous-time dynamic evaluation if it has the following properties:

- For all stopping times $\sigma, \Pi_{\sigma}$ is an $\mathcal{F}_{\sigma}$-conditional evaluation.

- Time-Consistency: For every $H \in L^{\infty}\left(\mathcal{F}_{T}\right)$ :

$$
\Pi_{\sigma}(H)=\Pi_{\sigma}\left(\Pi_{\tau}(H)\right) \text { for all stopping times } \sigma \leq \tau \text {. }
$$


In a dynamical context time-consistency is a natural assumption to glue together static risk measures. It means that the same risk is assigned to a financial position regardless of whether it is calculated over two time periods at once or in two steps backwards in time. For a general analysis of weaker notions of time-consistency see e.g. Roorda et al. (2005).

Remark 4.1 Alternative names for our definition of time-consistency would have been 'recursiveness' or 'tower property'. Note that in the literature often the following notion of time-consistency is used: if an asset $H_{1}$ is preferred to an asset $H_{2}$ under all possible scenarios at some time $\tau$ then $H_{1}$ should also have been preferred at every time $\sigma$ before $\tau$. Let us call the latter definition of time-consistency property (TC). Now if $\Pi$ is monotone it is well known that our notion of time-consistency is in fact equivalent to property (TC). Furthermore, since (TC) implies the monotonicity of $\Pi$, our notion of time-consistency includes (TC) as a special case, but it can also be applied to non-monotone evaluations.

Time-consistent evaluations have been discussed in continuous time by Peng (2004), Frittelli and Gianin (2004), Rosazza Gianin (2006), Delbaen (2006), Klöppel and Schweizer (2007), Bion-Nadal (2008), Bion-Nadal (2009), and Barrieu and El Karoui (2009). Duffie and Epstein (1992), Chen and Epstein (2002), and Maccheroni et al. (2006) deal with dynamic preferences using similar notions of time-consistency.

Given a dynamic evaluation $\left(\Pi_{\sigma}\right)$ we define $\Pi_{\sigma, \tau}$ to be equal to $\Pi_{\sigma}$ restricted to $L^{\infty}\left(\mathcal{F}_{\tau}\right)$, i.e., $\Pi_{\sigma, \tau}=\Pi_{\sigma} \mid L^{\infty}\left(\mathcal{F}_{\tau}\right)$. Next we will assume that the insurance process $Y$ a.s. jumps only at finitely many predictable time instances, say $0 \leq \tau_{1} \leq \tau_{2} \leq \ldots$ and there is no additional randomness added between the jumps, i.e., $\overline{\mathcal{F}}_{\tau_{i+1}-}^{Y}=\overline{\mathcal{F}}_{\tau_{i}}^{Y}$ for all $i$. Recall that a stopping time $\tau$ is predictable if there exists a sequence of stopping times $\tau^{n}<\tau$ such that $\tau^{n} \uparrow \tau$. Hitting times of continuous processes are predictable while jump times of Lévy processes (or more general, strong Markov Feller processes) are not. On the set where $Y$ does not jump at all we set $\tau_{1}=T{ }^{\ddagger}$ One example could be given by a setting in which the insurance process $Y$ is only updated at finitely many fixed time instances, $t_{1}<\ldots<t_{k}$. Another possibility could be that damages occur at unpredictable stopping times $\bar{\tau}_{i}$, but the amount of money the insurance will have to pay is not clear right away. Instead the insurance needs some additional time, say $\varepsilon>0$, to agree to a certain amount and to pay it out at $\bar{\tau}_{i}+\epsilon$, respectively.

We will use the following definition in a dynamic setting.

Definition 4.2 We will say that a conditional continuous-time evaluation $\left(\Pi_{\sigma}\right)_{\sigma \in[0, T]}$ is marketconsistent if for every stopping time $\sigma$ and every financial payoffs $H^{S} \in L^{\infty}\left(\overline{\mathcal{F}}_{T}^{S} \vee \mathcal{F}_{\sigma}\right)$ and $H \in L^{\infty}\left(\mathcal{F}_{T}\right)$

$$
\Pi_{\sigma}\left(H^{S}+H\right)=\mathbb{E}_{\mathcal{F}_{\sigma}}^{\mathbb{Q}}\left[H^{S}\right]+\Pi_{\sigma}(H) .
$$

Note that this definition of market-consistency coincides with the definition in the static case with $\mathcal{G}=\mathcal{F}_{\sigma}$ and $\overline{\mathcal{F}}^{S}=\overline{\mathcal{F}}_{T}^{S}$.

Now for every stopping time $\sigma$ we define $\tau_{\sigma}$ to be the time of the next jump after $\sigma$, i.e., $\tau_{\sigma}:=\inf \left\{t>\sigma \mid \Delta Y_{t}>0\right\} \wedge T$. Denote $\mathcal{F}_{\tau_{\sigma}}^{S}:=\overline{\mathcal{F}}_{\tau_{\sigma}}^{S} \vee \mathcal{F}_{\sigma}$. That is $\mathcal{F}_{\tau_{\sigma}}^{S}$ is the $\sigma$-algebra which includes all the information (of both stock and insurance process) up to time $\sigma$ and additionally the information of the stock process up to the time of the next jump of $Y$.

Theorem 4.3 Suppose that $S$ is continuous and the insurance process is as described above. Let $\left(\Pi_{\sigma}\right)_{0 \leq \sigma \leq T}$ be a time-consistent and market-consistent evaluation such that for every $\sigma$,

\footnotetext{
${ }^{\ddagger}$ Similarly we can set some $\tau_{i}(\omega)$ equal to $T$ if not all paths, $\left(Y_{t}(\omega)\right)_{t}$, have the same number of jumps.
} 
$\Pi_{\sigma}(\cdot)$ is continuous. Then for every stopping time $\sigma$, we have that $\Pi_{\sigma, \tau_{\sigma}}$ satisfies the marketlocal property. In particular, if $\left(\Pi_{\sigma}\right)$ is additionally assumed to be either monotone, p-norm bounded, or positively homogeneous then for every stopping time $\sigma$ there exists an $\mathcal{F}_{\tau_{\sigma}}^{S}$-conditional evaluation $\Pi_{\mathcal{F}_{\tau_{\sigma}}^{S}}: L^{\infty}\left(\mathcal{F}_{\tau_{\sigma}}\right) \rightarrow L^{\infty}\left(\mathcal{F}_{\tau_{\sigma}}^{S}\right)$ such that

$$
\Pi_{\sigma, \tau_{\sigma}}(H)=\mathbb{E}_{\mathcal{F}_{\sigma}}^{\mathbb{Q}}\left[\Pi_{\mathcal{F}_{\tau_{\sigma}}^{S}}(H)\right]
$$

This theorem shows in particular that in a setting where the agent just observes the insurance process at finitely many time instances, every market-consistent and time-consistent evaluation has to admit a representation of the form $\mathbb{E}_{\mathcal{F}_{\sigma}}^{\mathbb{Q}}\left[\Pi_{\mathcal{F}_{\tau_{\sigma}}^{S}}(H)\right]$ at every stopping time $\sigma$. In other words, an agent who wants to use a time-consistent and market-consistent evaluation has to apply a two step market evaluation.

Theorem 4.3 also yields the following corollary:

Corollary 4.4 Suppose that $S$ is continuous, and that the insurance process $Y$ a.s. jumps only at finitely many fixed time instances, say $0 \leq t_{1} \leq t_{2} \leq \ldots \leq t_{k}$, and $\overline{\mathcal{F}}_{t_{i+1}-}^{Y}=\overline{\mathcal{F}}_{t_{i}}^{Y}$. If $\left(\Pi_{\sigma}\right)_{0 \leq \sigma \leq T}$ is a time-consistent and market-consistent evaluation which is either monotone, $p$ norm bounded or positively homogeneous then for every $s \in[0, T]$ there exists an $\mathcal{F}_{t_{i}}^{S}$-conditional evaluation $\Pi_{\mathcal{F}_{t_{i}}^{S}}: L^{\infty}\left(\mathcal{F}_{t_{i}}\right) \rightarrow L^{\infty}\left(\mathcal{F}_{t_{i}}^{S}\right)$ such that

$$
\Pi_{s, t_{i}}(H)=\mathbb{E}_{\mathcal{F}_{s}}^{\mathbb{Q}}\left[\Pi_{\mathcal{F}_{t_{i}}^{S}}(H)\right],
$$

where $t_{i}$ is the next time instance after $s$ at which the insurance process jumps.

We have restricted our analysis to payoffs rather than payment streams. However, if $H$ is a discrete payment streams which pays cash amounts $\left(H_{\eta_{1}}, \ldots, H_{\eta_{k}}\right)$ at stopping times $\eta_{1}<\eta_{2}<$ $\ldots<\eta_{k}$, we could consider evaluations $\Pi_{\sigma}$ mapping payment streams, starting paying amounts from time $\sigma$ on, to $L^{\infty}\left(\mathcal{F}_{\sigma}\right)$. In this case time-consistency could be defined as

$$
\Pi_{\sigma}(H)=\Pi_{\sigma}\left(H I_{[0, \tau)}+\Pi_{\tau}\left(H I_{[\tau, T]}\right)\right) \text { for all } \sigma \leq \tau,
$$

see also Cheridito et al. (2006), or Jobert and Rogers (2008). By a proof analogue to the one for Theorem 4.3 one can then show that for an evaluation satisfying similar properties as above time-consistency and market-consistency entail

$$
\Pi_{\sigma, \tau_{\sigma}}\left(H I_{\left[\sigma, \tau_{\sigma}\right]}\right)=\mathbb{E}_{\mathcal{F}_{\sigma}}^{\mathbb{Q}}\left[\Pi_{\mathcal{F}_{\tau_{\sigma}}}\left(H I_{\left[\sigma, \tau_{\sigma}\right]}\right)\right]=\mathbb{E}_{\mathcal{F}_{\sigma}}^{\mathbb{Q}}\left[\Pi_{\mathcal{F}_{\tau_{\sigma}}}\left(H_{\tau_{\sigma}}\right)\right]+\mathbb{E}_{\mathcal{F}_{\sigma}}^{\mathbb{Q}}\left[\sum_{\sigma \leq \eta<\tau_{\sigma}} H_{\eta}\right]
$$

\section{Market-consistent evaluations in continuous time}

\subsection{Results on market-consistent BSDEs}

In a continuous time Brownian-Poisson setting we can provide examples of time-consistent and market-consistent evaluations by $g$-expectations. It is well known that $g$-expectations induce time-consistent evaluations, see for instance Peng (2004), Frittelli and Gianin (2004), Rosazza Gianin (2006), Bion-Nadal (2008), Barrieu and El Karoui (2009), or El Karoui and Ravanelli 
(2009). In this section we will give a complete characterization of $g$-expectations which are market-consistent.

Suppose the filtration $\mathcal{F}$ is generated by the following independent processes: an $n$-dimensional standard Brownian motion $W^{f}$, a $d$-dimensional standard Brownian motion $W$, and a Poisson random counting measure $N(d s, d x)$ defined on $[0, T] \times \mathbb{R} \backslash\{0\}$. We denote the corresponding compensator by

$$
\hat{N}(d s, d x)=\nu(d x) d s .
$$

We assume that the measure $\nu(d x)$ is non-negative and satisfies

$$
\int_{\mathbb{R} \backslash\{0\}}\left(|x|^{2} \wedge 1\right) \nu(d x)<\infty .
$$

Denote $\tilde{N}(d s, d x):=N(d s, d x)-\hat{N}(d s, d x)$.

Suppose that we have a bond $B$ with $B_{0}=1$ and $d B_{t}=r B_{t} d t$. We assume that the stock process $S=\left(S^{1}, \ldots, S^{n}\right)$ is given by

$$
d S_{t}^{i}=S_{t}^{i}\left(\mu^{i}\left(t, S_{t}\right) d t+\tilde{\sigma}^{i}\left(t, S_{t}\right) d W_{t}^{f}\right), \quad S_{0}^{i}=s_{0}^{i},
$$

with $s_{0}^{i}>0$ and $\mu^{i}:[0, T] \times \mathbb{R}^{n} \rightarrow \mathbb{R}$ and $\tilde{\sigma}^{i}:[0, T] \times \mathbb{R}^{n} \rightarrow \mathbb{R}^{n}$ for $i=1, \ldots, n$. Note that we used vector notation for the stochastic integral. Define the vector $\mu=\left(\mu^{i}\right)_{i=1, \ldots, n}$ taking values in $\mathbb{R}^{n}$. The rows of the matrix $\tilde{\sigma}:[0, T] \times \mathbb{R}^{n} \rightarrow \mathbb{R}^{n \times n}$ should be given by $\left(\tilde{\sigma}^{i}\right)_{i=1, \ldots, n}$. We assume that $\mu$ and $\tilde{\sigma}$ are uniformly bounded. Furthermore, $\tilde{\sigma}$ should be invertible, and uniformly elliptic, i.e., there exists $K_{1}, K_{2}>0$ such that $K_{1} I_{n} \preceq \tilde{\sigma} \tilde{\sigma}^{\top} \preceq K_{2} I_{n}$. Furthermore, we need standard measurability and Lipschitz continuity assumptions on $\mu$ and $\tilde{\sigma}$. Then it is well known that a solution $S$ for the SDE above exists and that the corresponding financial market, consisting of $(S, B)$, is complete. Generally payoffs can depend on $\left(W^{f}, W, \tilde{N}\right)$ and may not be replicable.

Let $\overline{\mathcal{F}}^{S}$ be the filtration generated by $S$. Denote the negative market price of uncertainty by $\theta_{t}=-\tilde{\sigma}^{-1}\left(t, S_{t}\right)\left(\mu\left(t, S_{t}\right)-r e\right)$, where $e$ is an $n$-dimensional vector consisting of ones. As in the sections before we will denote by $H$ hedgeable and unhedgeable discounted payoffs.

We will consider evaluations of $H$ given by the solutions of backward stochastic differential equations (BSDEs). Denote by $\mathcal{P}$ the predictable $\sigma$-algebra on the entire filtration. Let

$$
\mathcal{H}_{m}^{2}:=\left\{Z=\left(Z^{1}, \ldots, Z^{m}\right) \in \mathcal{P} \mid \mathbb{E}\left[\int_{0}^{T}\left|Z_{s}\right|^{2} d s\right]<\infty\right\}
$$

where we denote by $|\cdot|$ the Euclidean norm.

Let $\mathcal{S}^{2}$ be the space of all one-dimensional optional processes whose path-maximum is square integrable with respect to $\mathbb{P}$. Let $L^{2}(\nu(d x))$ be the space all $\mathcal{B}(\mathbb{R} \backslash\{0\})$-measurable functions mapping from $\mathbb{R} \backslash\{0\}$ to $\mathbb{R}$, which are square integrable with respect to $\nu$, where, as usual, two functions are identified if they are equal $\nu$ a.s. Define $L^{2}(\nu(d x) \times d \mathbb{P} \times d s)$ as all $\mathcal{P} \otimes \mathbb{B}(\mathbb{R} \backslash\{0\})$ measurable functions which are square-integrable with respect to $\nu(d x) \times d \mathbb{P} \times d s$. Now suppose that we have a suitably measurable function $g:[0, T] \times \Omega \times \mathbb{R}^{2} \times L^{2}(\nu(d x)) \rightarrow \mathbb{R}$.

A solution of the BSDE with driver $g\left(t, \omega, z^{f}, z, \tilde{z}\right)$ and terminal condition $H \in L^{\infty}\left(\mathcal{F}_{T}\right)$ is a quadruple of processes $\left(Y(H), Z^{f}, Z, \tilde{Z}\right) \in \mathcal{S}^{2} \times \mathcal{H}_{n}^{2} \times \mathcal{H}_{d}^{2} \times L^{2}(\nu(d x) \times d \mathbb{P} \times d s)$ such that

$d Y_{t}(H)=-g\left(t, Z_{t}^{f}, Z_{t}, \tilde{Z}_{t}\right) d t+Z_{t}^{f} d W_{t}^{f}+Z_{t} d W_{t}+\int_{\mathbb{R} \backslash\{0\}} \tilde{Z}_{t}(x) \tilde{N}(d t, d x)$ and $Y_{T}(H)=H$. 
Often BSDEs are also written in the following equivalent form:

$$
Y_{t}(H)=H+\int_{t}^{T} g\left(s, Z_{s}^{f}, Z_{s}, \tilde{Z}_{s}\right) d s-\int_{t}^{T} Z_{s}^{f} d W_{s}^{f}-\int_{t}^{T} Z_{s} d W_{s}-\int_{t}^{T} \int_{\mathbb{R} \backslash\{0\}} \tilde{Z}_{s}(x) \tilde{N}(d s, d x) .
$$

Since the terminal condition is given at maturity time $T$, BSDEs have to be computed backwards in time. As in many applications a terminal reward is specified and solutions of BSDEs satisfy a dynamic programming principle, BSDEs are often applied to solve problems in stochastic optimal control and mathematical finance, see apart from the papers mentioned above for instance El Karoui et al. (1997), Lazrak and Quenez (2003), Hamadène and Jeanblanc (2007), and Porchet et al. (2009); or for the discrete time case Madan et al. (2010).

Subsequently, we will always assume that the BSDE which we consider has a unique solution. This is for instance the case if $g(t, 0,0,0)$ is in $L^{2}(d \mathbb{P} \times d t)$, and $g$ is uniformly Lipschitz continuous; that is there exists $K>0$ such that

$$
\left|g\left(t, z_{1}^{f}, z_{1}, \tilde{z}_{1}\right)-g\left(t, z_{0}^{f}, z_{0}, \tilde{z}_{0}\right)\right| \leq K\left(z_{1}^{f}-z_{0}^{f}|+| z_{1}-z_{0} \mid+\sqrt{\int_{\mathbb{R} \backslash\{0\}}\left|\tilde{z}_{1}(x)-\tilde{z}_{0}(x)\right|^{2} \nu(d x)}\right)
$$

see for instance Royer (2006) and the reference therein.

Example 5.1 Let $H$ be a bounded payoff and define $Y_{t}(H)=\mathbb{E}_{\mathcal{F}_{t}}[H]$. Then by the martingale representation theorem (see e.g. Jacod and Shiryaev (1987), Sec. 3, Theorem 4.34) there exist predictable square-integrable processes $Z^{f}, Z$ and $\tilde{Z}$ such that $Y$ satisfies

$$
d Y_{t}(H)=Z_{t}^{f} d W_{t}^{f}+Z_{t} d W_{t}+\int_{\mathbb{R} \backslash\{0\}} \tilde{Z}_{t}(x) \tilde{N}(d t, d x) \text { and } Y_{T}(H)=H .
$$

This is the simplest BSDE with $g=0$.

Hence, a conditional expectation may be seen as a BSDE with $g=0$. This is why BSDEs are being referred to as $g$-expectations. The name should express that a BSDE may be viewed as generalized (usually non-linear) conditional expectation with an additional drift.

Example 5.2 Let $H$ be a bounded payoff and define $Y_{t}(H)=\mathbb{E}_{\mathcal{F}_{t}}^{\mathbb{Q}}[H]$. Then by the martingale representation theorem and by the Girsanov theorem $Y(H)$ satisfies

$$
d Y_{t}(H)=-\theta_{t} Z_{t}^{f} d t+Z_{t}^{f} d W_{t}^{f}+Z_{t} d W_{t}+\int_{\mathbb{R} \backslash\{0\}} \tilde{Z}_{t}(x) \tilde{N}(d t, d x) \text { and } Y_{T}(H)=H .
$$

This is a linear BSDE with $g\left(t, z^{f}, z, \tilde{z}\right)=\theta_{t} z^{f}$.

Subsequently, we will write

$$
\mathcal{E}_{t}^{g}(H)=Y_{t}(H)
$$

In a Markovian setting $g$-expectations correspond to semi-linear parabolic PDEs (or PIDEs in the case of jumps), see for instance El Karoui et al. (1997) in a Brownian setting (see Barles et al. (1997) in the case of jumps).

It may be seen that if $g$ is convex and $g(t, 0,0,0)=0$ then the evaluation defined by

$$
\Pi_{\sigma}(H):=\mathcal{E}_{\sigma}^{g}(H)
$$


is normal, monotone, cash invariant, convex, time-consistent and satisfies the local property. Hence, $g$-expectations give us an abundance of time-consistent, continuous-time evaluations. There are also certain sufficient conditions under which, in a Brownian filtration, a timeconsistent evaluation is induced as the solution of a $g$-expectation, see Coquet et al. (2002).

The following theorem gives a complete characterization of market-consistent evaluations given by $g$-expectations:

Theorem 5.3 A g-expectation is market-consistent if and only if $g\left(t, z^{f}, z, \tilde{z}\right)-\theta_{t} z^{f}$ does not depend on $z^{f} d \mathbb{P} \times d t$ a.s.

\subsection{Examples of market-consistent BSDEs}

To get an interpretation of $g$ we will consider some examples. The next proposition gives a dynamic market-consistent extension of the exponential principle. It follows directly from Theorem 5.3 above and Theorem 2 in Morlais (2010). (Morlais (2010) also allows for a hedging set $\mathcal{C}$ and an initial capital amount $x$. In our case this becomes $\mathcal{C}=\{0\}$ and $x=0$.)

Proposition 5.4 Define the evaluation $\Pi$ as the solution of the BSDE with driver function

$$
g\left(t, z^{f}, z, \tilde{z}\right)=\theta_{t} z^{f}+\frac{1}{2 \gamma}|z|^{2}+\gamma \int_{\mathbb{R} \backslash\{0\}}\left[\exp \left\{\frac{\tilde{z}(x)}{\gamma}\right\}-\frac{\tilde{z}(x)}{\gamma}-1\right] \nu(d x) .
$$

Then (i) $\Pi$ is market-consistent, and (ii) for pure insurance risk (i.e., terminal conditions independent of $S) \Pi$ corresponds to the exponential principle from Example 2.3.

Other examples of the driver function $g$ can be obtained by looking at one-period evaluations in discrete time defined recursively. Namely, suppose that we have an equi-spaced time grid $I=\{0, h, 2 h, \ldots, T\}$ where we assumed without loss of generality that $T$ is a multiple of $h$. The filtration $\left(\mathcal{F}_{i h}\right)_{i=0,1, \ldots, T / h}$ is generated by $\left(W_{i h}^{f}, W_{i h}, \tilde{N}((0, i h], d x)\right)_{i=1, \ldots, T / h}$. Define $S_{0}^{h, j}=s_{0}^{j}$, $S_{(i+1) h}^{h, j}=S_{i h}^{h, j}\left(1+\mu^{j}\left(i h, S_{i h}^{h}\right) h+\tilde{\sigma}^{j}\left(i h, S_{i h}^{h}\right) \Delta W_{(i+1) h}^{f}\right)$ for $i=1, \ldots, T / h$ and $j=1, \ldots, n$, and $S^{h}=\left(S^{h, 1}, \ldots, S^{h, n}\right)$. Denote further $\mathcal{F}_{(i+1) h}^{S^{h}}=\overline{\mathcal{F}}_{(i+1) h}^{S^{h}} \vee \mathcal{F}_{i h}$, where $\overline{\mathcal{F}}^{S^{h}}$ is the filtration generated by $S^{h}$. In other words $\mathcal{F}^{S^{h}}$ is the information of the (discrete-time) stock process together with the previous values of the insurance process. Let $\mathbb{Q}^{h}$ be the measure (with $\overline{\mathcal{F}}^{S^{h}}$-measurable density) such that $\Delta W_{(i+1) h}^{f, *}:=\Delta W_{(i+1) h}^{f}-\theta_{i h} h$ is a martingale.

Now we can use evaluations from our Examples 3.4 over one period and glue them together recursively. Using our two step procedures for the one-periodic evaluations could be natural, in particular, if the stock process can be observed before the insurance process. Suppose for instance for a moment that we are at time $i h$ and the stock process, $S^{h}$, can be observed at time $\left(i+\frac{1}{2}\right) h$, whereas the insurance process is revealed after the stock process at time $(i+1) h$. Since data from the financial market can be observed almost continuously, while data from insurance companies are typically observed less frequently, this may not be an unreasonable assumption, see also Section 4. Of course our insurance process will possibly be effected by the financial market through its correlation to $W^{f}$. However, it will not be completely predictable due to its dependance on the jumps and $W$. In this situation if an evaluation $\left(\Pi_{\sigma}\right)_{\sigma \in[0, T]}$ is time-consistent, then market-consistency would imply that for the aggregated evaluation $\left(\Pi_{i h}\right)_{i=0,1, \ldots, T / h}$ we have

$$
\Pi_{i h}\left(H_{(i+1) h}\right)=\Pi_{i h}\left(\Pi_{\left(i+\frac{1}{2}\right) h}\left(H_{(i+1) h}\right)\right)=\mathbb{E}_{\mathcal{F}_{i h}}^{\mathbb{Q}^{h}}\left[\Pi_{\left(i+\frac{1}{2}\right) h}\left(H_{(i+1) h}\right)\right] .
$$


The last equation holds by market-consistency since $\Pi_{\left(i+\frac{1}{2}\right) h}\left(H_{(i+1) h}\right)$, is $\mathcal{F}_{\left(i+\frac{1}{2}\right) h}^{S^{h}}=\overline{\mathcal{F}}_{\left(i+\frac{1}{2}\right) h}^{S^{h}} \vee$ $\mathcal{F}_{i h}$-measurable. Therefore, the setting outlined above would indeed give rise to applying a two step market evaluation at time-instances $i h$, to evaluate payoffs up to time $(i+1) h$. We will calculate one example explicitly by considering at time instances $i h$ the mean-variance two step market evaluation

$$
\Pi_{i h,(i+1) h}^{v}\left(H_{(i+1) h}\right)=\mathbb{E}_{\mathcal{F}_{i h}^{h}}^{\mathbb{Q}^{h}}\left[\mathbb{E}_{\mathcal{F}_{(i+1) h}^{S h}}\left[H_{(i+1) h}\right]+\frac{1}{2} \alpha \operatorname{Var}_{\mathcal{F}_{(i+1) h}^{S h}}\left[H_{(i+1) h}\right]\right], \quad \alpha \geq 0 .
$$

To obtain a multi-period evaluation $\left(\Pi_{i h}^{h}\right)_{i=0,1, \ldots, T / h}$ on the whole filtration with $\Pi_{i h}^{h}$ : $L^{\infty}\left(\mathcal{F}_{T}\right) \rightarrow L^{\infty}\left(\mathcal{F}_{i h}\right)$ we will define $\Pi^{h}$ recursively by setting

$$
\Pi_{T}^{h}(H)=H \text { and } \Pi_{i h}^{h}(C)=\Pi_{\mathcal{F}_{i h}}^{v}\left(\Pi_{(i+1) h}^{h}(H)\right) \text { for } i=0,1, \ldots, T / h-1 .
$$

The following proposition is proved in the appendix:

Proposition 5.5 Suppose that the evaluation $\Pi^{h}$ is constructed by (5.1), i.e., using locally the Mean-Variance two step market evaluation. Then for every terminal payoff $H \in L^{\infty}\left(\mathcal{F}_{T}\right)$ there exists predictable $\left(Z^{h, f}, Z^{h}, \tilde{Z}^{h}\right)$ and a martingale $\left(L_{i h}^{h}\right)_{i}$ orthogonal (under $\left.\mathbb{Q}^{h}\right)$ to $\left(W_{i h}^{f}, W_{i h}, \tilde{N}((0, i h], d x)\right)_{i}$ such that for all $i$ we have

$$
\begin{aligned}
\Pi_{i h}^{h}(H) & =H+\sum_{j=i}^{T / h-1}\left(\left[\theta_{j h} Z_{j h}^{h, f}+\frac{\alpha}{2}\left(\left|Z_{j h}^{h}\right|^{2}+\int_{\mathbb{R} \backslash\{0\}}\left|\tilde{Z}_{j h}^{h}(x)\right|^{2} \nu(d x)\right)\right] h\right. \\
& \left.+\frac{\alpha}{2} \mathbb{E}_{\mathcal{F}_{j h}^{h}}^{\mathbb{Q}^{h}}\left[\left(\Delta L_{(j+1) h}^{h}-\mathrm{E}_{\mathcal{F}_{(j+1) h}^{S h}}\left[\Delta L_{(j+1) h}^{h}\right]\right)^{2}\right]\right)-\sum_{j=i}^{T / h-1} Z_{j h}^{h, f} \Delta W_{(j+1) h}^{f} \\
& -\sum_{j=i}^{T / h-1} Z_{j h}^{h} \Delta W_{(j+1) h}-\sum_{j=i}^{T / h-1} \int_{\mathbb{R} \backslash\{0\}} \tilde{Z}_{j h}^{h}(x) \tilde{N}((j h,(j+1) h], d x)-\left(L_{T}^{h}-L_{i h}^{h}\right) .
\end{aligned}
$$

In particular, $\Pi^{h}$ satisfies a discrete-time BSDE.

From Proposition 5.5 we may infer that

$$
\begin{array}{r}
\mathbb{E}_{\mathcal{F}_{j h}}\left[\Delta \Pi_{(j+1) h}^{h}(H)\right]=-\left[\theta_{j h} Z_{j h}^{h, f}+\frac{\alpha}{2}\left(\left|Z_{j h}^{h}\right|^{2}+\int_{\mathbb{R} \backslash\{0\}}\left|\tilde{Z}_{j h}^{h}(x)\right|^{2} \nu(d x)\right)\right] h \\
-\frac{\alpha}{2} \mathbb{E}_{\mathcal{F}_{j h}^{h}}\left[\left(\Delta L_{(j+1) h}^{h}-\mathrm{E}_{\mathcal{F}_{(j+1) h}^{S h}}\left[\Delta L_{(j+1) h}^{h}\right]\right)^{2}\right] .
\end{array}
$$

Note that the orthogonal martingale terms $\Delta L_{(j+1) h}^{h}$ arise because the discretized Brownian Motions do not have the representation property. However, the continuous time Brownian motions and the Poisson random measure do have the representation property. Therefore, if we ignore the $L^{h}$ then an analogous infinitesimal way of charging the risk in continuous time would be an evaluation which satisfies

$$
\mathbb{E}_{\mathcal{F}_{t}}\left[d \Pi_{t}(H)\right]=-\left[\theta_{t} Z_{t}^{f}+\frac{\alpha}{2}\left(\left|Z_{t}\right|^{2}+\int_{\mathbb{R} \backslash\{0\}}\left|\tilde{Z}_{t}(x)\right|^{2} \nu(d x)\right)\right] d t .
$$

This corresponds to an evaluation given by the solution of a BSDE with driver function

$$
g(t, z, \tilde{z})=\theta_{t} z^{f}+\frac{\alpha}{2}\left(|z|^{2}+\int_{\mathbb{R} \backslash\{0\}}|\tilde{z}(x)|^{2} \nu(d x)\right) .
$$


Remark 5.6 The analogy stated above only corresponds to the local way of charging risk. A global correspondence of charging risk would involve proving a convergence result for the whole path. In the case that the driver function is Lipschitz continuous it can actually be shown in a purely Brownian setting that after an appropriate scaling the whole path of the discrete time evaluations converges to the corresponding solution of the BSDE; see Stadje (2010). However, in Proposition 5.5. the driver function is quadratic in $z$. In this case already in the purely Brownian setting an extension by convergence may not always be possible. Cheridito and Stadje (2010) give an example which shows that in a setting where the discrete time filtration is generated by a Bernoulli random walk it may happen that the discrete time $H^{n}$ are uniformly bounded and converge to $H \in L^{\infty}(\mathcal{F})$ but the discrete time evaluations explode.

\section{Summary \& Conclusions}

In this paper we have studied the extension of standard actuarial principles in time-consistent and market-consistent directions by introducing a new market-consistent evaluation procedure which we call 'two step market evaluation.' On the one hand, two step market evaluations sometimes arise when an agent starts with an evaluation that is not market-consistent, such as the Average Value at Risk or the Exponential Premium principle, and then engages in hedging. On the other hand, market-consistent evaluations can also be defined directly by applying a standard evaluation technique, conditional on the stock process. In this case the structure of many standard evaluation techniques can be preserved.

We have shown that two step market evaluations are invariant if the stock is taken as a numeraire. In Theorem 3.8 and Theorem 3.9 a complete axiomatic characterization for two step market evaluations is provided. Moreover, we have proved that in a dynamic setting with a continuous stock prices process and an insurance process being revealed at predictable times every evaluation which is time-consistent and market-consistent is a two step market evaluation up to the next predictable time, which gives a strong argument for their use. We have also characterized market-consistency in terms of $g$-expectations and studied the extension of the mean-variance and the exponential principle to continuous time, in a setting with jumps. Our analysis shows that two step evaluations can provide a useful, computationally tractable tool for market-consistent valuations.

\section{A Appendix: Technical Material \& Proofs}

\section{A.1 Background to the Essential Supremum}

The first part of the appendix is basically a summary of the definitions and results given in A.5 Föllmer and Schied (2004). Consider a family of random variables $M$ on a given probability space $(\Omega, \overline{\mathcal{F}}, \mathbb{P})$. Now if $M$ is countable then $Z^{*}(\omega)=\sup _{Z \in M} Z(\omega)$ is also measurable. However, measurability is not guaranteed if $M$ is uncountable. Even if the pointwise supremum is measurable it might not be the right concept when we focus on a.s. properties. For instance if $\mathbb{P}$ is the Lesbegue measure on $\Omega=[0,1]$ and $M=\left\{I_{\{x\}} \mid 0 \leq x \leq 1\right\}$ then $\sup _{Z \in M} Z=1$ while $Z=0$ a.s. for all $Z \in M$. This suggest the following notion of an essential supremum defined in terms of almost sure inequalities. This result can be found as Theorem A.32 in Föllmer and Schied (2004). 
Theorem A.1 Let $M$ be any set of random variables on $(\Omega, \overline{\mathcal{F}}, \mathbb{P})$.

(a) There exists a random variable $Z^{*}$ such that

$$
Z^{*} \geq Z \mathbb{P} \text {-a.s. for all } Z \in M \text {. }
$$

Moreover, $Z^{*}$ is a.s. unique in the following sense: Any other random variable $\hat{Z}$ with property (A) satisfies $\hat{Z} \geq Z^{*} \mathbb{P}$-a.s.

(b) Suppose that $M$ is directed upwards, i.e., for $Z_{1}, Z_{2} \in M$ there exists $Z_{3} \in M$ with $Z_{3} \geq \max \left(Z_{1}, Z_{2}\right)$. Then there exists an increasing sequence $Z_{1} \leq Z_{2} \leq \ldots$ in $M$ such that $Z^{*}=\lim _{n} Z_{n} \mathbb{P}$-a.s.

Definition A.2 The random variable $Z^{*}$ in the the theorem above is called the essential supremum of $M$ and we write:

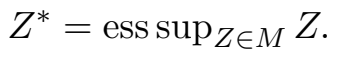

We define the essential infimum similarly.

If the probability space is finite the essential supremum corresponds to the pointwise supremum taken over all atoms.

Lemma A.3 If $M$ satisfies the concatenation property, i.e., for every $A \in \overline{\mathcal{F}}$ and $Z_{1}, Z_{2} \in M$ we have that $Z_{1} I_{A}+Z_{2} I_{A^{c}} \in M$, then $M$ is directed upwards.

Proof. Define

$$
Z^{*}=Z_{1} I_{\left\{Z_{1} \geq Z_{2}\right\}}+Z_{2} I_{\left\{Z_{1}<Z_{2}\right\}}
$$

By the concatenation property $Z^{*} \in M$, and by definition $Z^{*} \geq \max \left(Z_{1}, Z_{2}\right)$.

\section{A.2 Proofs of the results in Section 3.1}

Proof of Proposition 3.3. (i) $\Rightarrow$ (ii) By (2.2) we have that

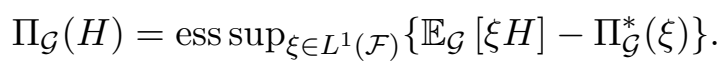

Furthermore,

$$
\begin{aligned}
\Pi_{\mathcal{G}}^{*}(\xi) & =\operatorname{ess}_{\sup _{H \in L^{\infty}(\mathcal{F})}}\left\{\mathbb{E}_{\mathcal{G}}[\xi H]-\Pi_{\mathcal{G}}(H)\right\} \\
& \geq \operatorname{ess}_{\sup _{H \in L^{\infty}\left(\mathcal{F}^{S}\right)}}\left\{\mathbb{E}_{\mathcal{G}}[\xi H]-\Pi_{\mathcal{G}}(H)\right\} \\
& =\operatorname{ess}_{\sup _{H \in L^{\infty}\left(\mathcal{F}^{S}\right)}}\left\{\mathbb{E}_{\mathcal{G}}\left[\mathbb{E}_{\mathcal{F}^{S}}[\xi] H\right]-\Pi_{\mathcal{G}}(H)\right\}
\end{aligned}
$$

The last term in (A.3) is the dual of $\Pi_{\mathcal{G}}$ restricted to $\mathcal{F}^{S}$ evaluated at $\mathbb{E}_{\mathcal{F}^{S}}[\xi]$. Now by assumption $\Pi_{\mathcal{G}}\left(H^{S}\right)=\mathbb{E}^{\mathbb{Q}_{\mathcal{G}}}\left[H^{S}\right]$ is linear. Thus, its dual penalty function, $\left(\Pi_{\mathcal{G}} \mid \mathcal{F}^{S}\right)^{*}: L^{1}\left(\mathcal{F}^{S}\right) \rightarrow$ $\mathbb{R} \cup\{\infty\}$, must be equal to the indicator function which is zero if the input argument is $\frac{d \mathbb{Q}_{\mathcal{G}}}{d \mathbb{P}_{\mathcal{G}}}$, and infinity else. But then, by the inequality above, $\Pi_{\mathcal{G}}^{*}(\xi)$ must be equal to infinity as well if $\mathbb{E}_{\mathcal{F}^{S}}[\xi] \neq \frac{d \mathbb{Q}_{\mathcal{G}}}{d \mathbb{P}_{\mathcal{G}}}$. Thus, it is sufficient to consider $\xi$ of the form $\xi=\frac{d \mathbb{Q}_{\mathcal{G}}}{d \mathbb{P}_{\mathcal{G}}} Z$ for a $Z:=\frac{d \mathbb{Q}_{\mathcal{G}}}{d \mathbb{P}_{\mathcal{G}}} \in \mathcal{Q}_{\mathcal{F} S}$. Defining for $Z \in \mathcal{Q}_{\mathcal{F} S}, c_{\mathcal{G}}(Z)=\Pi_{\mathcal{G}}^{*}\left(\frac{d \mathbb{Q}_{\mathcal{G}}}{d \mathbb{P}_{\mathcal{G}}} Z\right)$, we have indeed that

$$
\begin{aligned}
& \Pi_{\mathcal{G}}(H)=\operatorname{ess}_{\sup _{\xi \in L^{1}(\mathcal{F})}}\left\{\mathbb{E}_{\mathcal{G}}[\xi H]-\Pi_{\mathcal{G}}^{*}(\xi)\right\}
\end{aligned}
$$

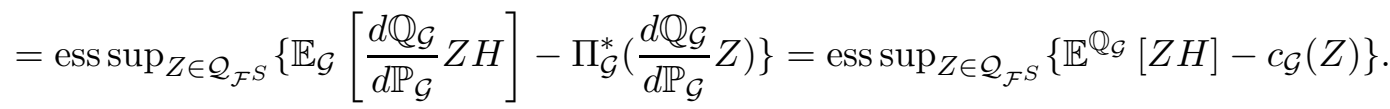


(ii) $\Rightarrow$ (iii): It is by (ii)

$$
\begin{aligned}
& \Pi_{\mathcal{G}}\left(H^{S}+H\right)=\operatorname{ess}_{\sup _{Z \in \mathcal{Q}_{\mathcal{F}}}}\left\{\mathbb{E}_{\mathcal{G}}\left[\frac{d \mathbb{Q}_{\mathcal{G}}}{d \mathbb{P}_{\mathcal{G}}} Z\left(H^{S}+H\right)\right]-c_{\mathcal{G}}(Z)\right\} \\
& =\operatorname{ess} \sup _{Z \in \mathcal{Q}_{\mathcal{F}} S}\left\{\mathbb{E}_{\mathcal{G}}\left[\frac{d \mathbb{Q}_{\mathcal{G}}}{d \mathbb{P}_{\mathcal{G}}} Z H^{S}\right]+\mathbb{E}_{\mathcal{G}}\left[\frac{d \mathbb{Q}_{\mathcal{G}}}{d \mathbb{P}_{\mathcal{G}}} Z H\right]-c_{\mathcal{G}}(Z)\right\} \\
& =\operatorname{ess}_{\sup _{Z \in \mathcal{Q}_{\mathcal{F}}}}\left\{\mathbb{E}_{\mathcal{G}}\left[\mathbb{E}_{\mathcal{F} S}\left[\frac{d \mathbb{Q}_{\mathcal{G}}}{d \mathbb{P}_{\mathcal{G}}} Z H^{S}\right]\right]+\mathbb{E}_{\mathcal{G}}\left[\frac{d \mathbb{Q}_{\mathcal{G}}}{d \mathbb{P}_{\mathcal{G}}} Z H\right]-c_{\mathcal{G}}(Z)\right\} \\
& =\operatorname{ess} \sup _{Z \in \mathcal{Q}_{\mathcal{F} S}}\left\{\mathbb{E}_{\mathcal{G}}\left[\frac{d \mathbb{Q}_{\mathcal{G}}}{d \mathbb{P}_{\mathcal{G}}} H^{S} \mathbb{E}_{\mathcal{F} S}[Z]\right]+\mathbb{E}_{\mathcal{G}}\left[\frac{d \mathbb{Q}_{\mathcal{G}}}{d \mathbb{P}_{\mathcal{G}}} Z H\right]-c_{\mathcal{G}}(Z)\right\} \\
& =\operatorname{ess}_{\sup _{Z \in \mathcal{Q}_{\mathcal{F}} S}}\left\{\mathbb{E}_{\mathcal{G}}\left[\frac{d \mathbb{Q}_{\mathcal{G}}}{d \mathbb{P}_{\mathcal{G}}} H^{S}\right]+\mathbb{E}_{\mathcal{G}}\left[\frac{d \mathbb{Q}_{\mathcal{G}}}{d \mathbb{P}_{\mathcal{G}}} Z H\right]-c_{\mathcal{G}}(Z)\right\} \\
& =\mathbb{E}_{\mathcal{G}}\left[\frac{d \mathbb{Q}_{\mathcal{G}}}{d \mathbb{P}_{\mathcal{G}}} H^{S}\right]+\operatorname{ess}^{\sup _{Z \in \mathcal{Q}_{\mathcal{F}}}}\left\{\mathbb{E}_{\mathcal{G}}\left[\frac{d \mathbb{Q}_{\mathcal{G}}}{d \mathbb{P}_{\mathcal{G}}} Z H\right]-c_{\mathcal{G}}(Z)\right\}=\mathbb{E}^{\mathbb{Q}_{\mathcal{G}}}\left[H^{S}\right]+\Pi_{\mathcal{G}}(H),
\end{aligned}
$$

where we have used in the fourth equation that $d \mathbb{Q}_{\mathcal{G}} / d \mathbb{P}_{\mathcal{G}}$ and $H^{S}$, by assumption, are $\mathcal{F}^{S}$ measurable. In the fifth equation we have used that $\mathbb{E}_{\mathcal{F}^{S}}[Z]=1$. This proves (ii) $\Rightarrow$ (iii). The direction (iii) $\Rightarrow$ (i) is clear using that by normalization $\Pi_{\mathcal{G}}(0)=0$.

Finally let us show that if $\Pi_{\mathcal{G}}$ is additionally assumed to be monotone, then marketconsistency is equivalent to any payoff being evaluated between its super- and sub-replication price. Clearly, if any payoff is evaluated between its super- and sub-replication price then for any $H^{S} \in L^{\infty}\left(\mathcal{F}^{S}\right)$ we must have that $\Pi_{\mathcal{G}}\left(H^{S}\right)=\mathbb{E}^{\mathbb{Q}}{ }_{\mathcal{G}}\left[H^{S}\right]$, as the financial market is assumed to be complete. Hence, by the direction (i) $\Rightarrow$ (iii) shown above, indeed $\Pi_{\mathcal{G}}$ is market-consistent. On the other hand if $\Pi_{\mathcal{G}}$ is market-consistent and monotone then by standard duality results the penalty function in (ii) must have a domain in $\mathcal{Q}_{\mathcal{F} S}^{+}$. Also note that the set defined by $M:=\frac{d \mathbb{Q}_{\mathcal{G}}}{d \mathbb{P}_{\mathcal{G}}} \mathcal{Q}_{\mathcal{F} S}^{+}=\left\{\frac{d \mathbb{Q}_{\mathcal{G}}}{d \mathbb{P}_{\mathcal{G}}} Z \mid Z \in \mathcal{Q}_{\mathcal{F} S}^{+}\right\}$is equal to the set of all local martingale measures $\mathcal{M}_{\mathcal{G}}$. (Actually for our proof we only need that $M \subset \mathcal{M}_{\mathcal{G}}$.) This yields that

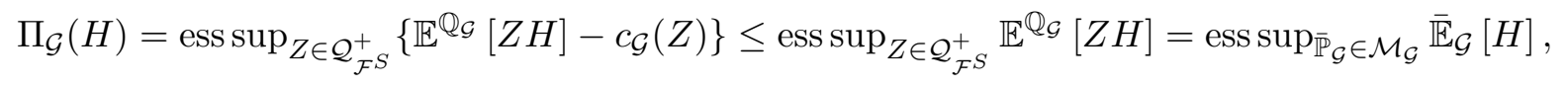

where we have used in the first equality that (ii) holds as $\Pi_{\mathcal{G}}$ is market-consistent. In the inequality we applied that $c_{\mathcal{G}} \geq 0$. In the last equality we used that $M=\mathcal{M}_{\mathcal{G}}$. Hence, indeed $\Pi_{\mathcal{G}}(H)$ is smaller than the super-replication price of $H$. To show that $\Pi_{\mathcal{G}}(H)$ is greater than the sub-replication price, note that as $c$ is a penalty function we must have $\operatorname{ess} \inf _{Z \in \mathcal{Q}_{\mathcal{F} S}^{+}} c_{\mathcal{G}}(Z)=0$. Now clearly, $\mathcal{Q}_{\mathcal{F} S}^{+}$satisfies the concatenation property from Lemma A.3. Thus, there exists a sequence $Z^{n} \in \mathcal{Q}_{\mathcal{F} S}^{+}$such that a.s. $\lim _{n} c_{\mathcal{G}}\left(Z^{n}\right)=0$. This entails that

$$
\begin{aligned}
& \Pi_{\mathcal{G}}(H)=\operatorname{ess}_{\sup _{Z \in \mathcal{Q}_{\mathcal{F}}^{+}}}\left\{\mathbb{E}^{\mathbb{Q}_{\mathcal{G}}}[Z H]-c_{\mathcal{G}}(Z)\right\} \\
& \geq \lim _{n}\left\{\mathbb{E}^{\mathbb{Q}_{\mathcal{G}}}\left[Z^{n} H\right]-c_{\mathcal{G}}\left(Z^{n}\right)\right\} \\
& \geq \liminf _{n} \mathbb{E}^{\mathbb{Q}_{\mathcal{G}}}\left[Z^{n} H\right]+\liminf _{n}-c_{\mathcal{G}}\left(Z^{n}\right)=\liminf _{n} \mathbb{E}^{\mathbb{Q}_{\mathcal{G}}}\left[Z^{n} H\right] \geq \operatorname{essinf}_{\overline{\mathbb{P}}_{\mathcal{G}} \in \mathcal{M}_{\mathcal{G}}} \overline{\mathbb{E}}_{\mathcal{G}}[H] .
\end{aligned}
$$

Hence, $\Pi_{\mathcal{G}}(H)$ is greater than the sub-replication price. The proposition is proved.

Theorems 3.10 and 3.11 may be seen as versions of the Radon-Nikodyn theorem with a non-linear part $\Pi_{\mathcal{F}^{S}}$ and without assumptions like monotonicity or continuity. We will need the following Lemma. Its proof is straight-forward doing an induction over $r$. 
Lemma A.4 Suppose that $\Pi_{\mathcal{G}}$ satisfies market-consistency and the local property. Then for disjoint sets $C_{1}, \ldots, C_{r} \in \mathcal{F}^{S}$ and payoffs $H_{1}, \ldots, H_{r} \in L^{\infty}(\mathcal{F})$, we have

$$
\Pi_{\mathcal{G}}\left(H_{1} I_{C_{1}}+\ldots+H_{r} I_{C_{r}}\right)=\Pi_{\mathcal{G}}\left(H_{1} I_{C_{1}}\right)+\ldots+\Pi_{\mathcal{G}}\left(H_{r} I_{C_{r}}\right) .
$$

The next lemma will also be useful.

Lemma A.5 $A \overline{\mathcal{G}}$-conditional evaluation satisfies $\Pi_{\overline{\mathcal{G}}}\left(I_{A} H\right)=I_{A} \Pi_{\overline{\mathcal{G}}}(H)$ for every $A \in \overline{\mathcal{G}}$.

Proof. It is

$$
\Pi_{\overline{\mathcal{G}}}\left(I_{A} H\right)=\Pi_{\overline{\mathcal{G}}}\left(I_{A} H+I_{A^{c}} 0\right)=I_{A} \Pi_{\overline{\mathcal{G}}}(H)+I_{A^{c}} \Pi_{\overline{\mathcal{G}}}(0)=I_{A} \Pi_{\overline{\mathcal{G}}}(H),
$$

by normalization and the local property of $\Pi_{\overline{\mathcal{G}}}$.

For the proof of Theorem 3.10 we will also need the following lemma:

Lemma A.6 In the setting of Theorem 3.10, the set $M^{\prime}$ defined by (3.4) has the concatenation property in the sense that $Z_{1}, Z_{2} \in M^{\prime}$ implies that for any $A \in \mathcal{F}^{S}$ we have that $Z_{1} I_{A}+Z_{2} I_{A^{c}} \in$ $M^{\prime}$. In particular, $I_{A} M^{\prime}+I_{A^{c}} M^{\prime}:=\left\{Z_{1} I_{A}+Z_{2} I_{A^{c}} \mid Z_{1} \in M^{\prime}, Z_{2} \in M^{\prime}\right\}=M^{\prime}$.

Proof. For $Z_{1}, Z_{2} \in M^{\prime}$ we have

$$
\begin{aligned}
& \Pi_{\mathcal{G}}^{*}\left(\frac{d \mathbb{Q}_{\mathcal{G}}}{d \mathbb{P}_{\mathcal{G}}}\left(Z_{1} I_{A}+Z_{2} I_{A^{c}}\right)\right) \\
& =\operatorname{ess}_{\sup } \operatorname{sL}^{\infty}(\mathcal{F})\left\{\mathbb{E}_{\mathcal{G}}\left[\frac{d \mathbb{Q}_{\mathcal{G}}}{d \mathbb{P}_{\mathcal{G}}}\left(Z_{1} I_{A}+Z_{2} I_{A^{c}}\right) H\right]-\Pi_{\mathcal{G}}(H)\right\}
\end{aligned}
$$

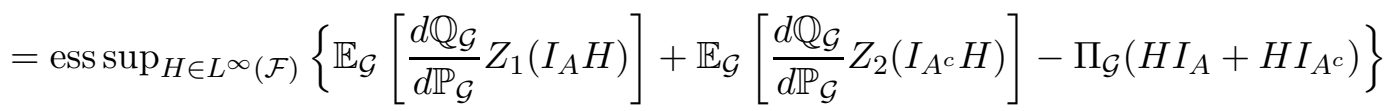

$$
\begin{aligned}
& =\operatorname{ess} \sup _{H I_{A}, H I_{A^{c}} \in L^{\infty}(\mathcal{F})}\left\{\mathbb{E}_{\mathcal{G}}\left[\frac{d \mathbb{Q}_{\mathcal{G}}}{d \mathbb{P}_{\mathcal{G}}} Z_{1}\left(I_{A} H\right)\right]-\Pi_{\mathcal{G}}\left(H I_{A}\right)\right. \\
& \left.+\mathbb{E}_{\mathcal{G}}\left[\frac{d \mathbb{Q}_{\mathcal{G}}}{d \mathbb{P}_{\mathcal{G}}} Z_{2}\left(I_{A^{c}} H\right)\right]-\Pi_{\mathcal{G}}\left(H I_{A^{c}}\right)\right\} \\
& =\operatorname{ess} \sup _{H I_{A} \in L^{\infty}(\mathcal{F})}\left\{\mathbb{E}_{\mathcal{G}}\left[\frac{d \mathbb{Q}_{\mathcal{G}}}{d \mathbb{P}_{\mathcal{G}}} Z_{1}\left(H I_{A}\right)\right]-\Pi_{\mathcal{G}}\left(H I_{A}\right)\right\} \\
& +\operatorname{ess} \sup _{H I_{A^{c}} \in L^{\infty}(\mathcal{F})}\left\{\mathbb{E}_{\mathcal{G}}\left[\frac{d \mathbb{Q}_{\mathcal{G}}}{d \mathbb{P}_{\mathcal{G}}} Z_{2}\left(H I_{A^{c}}\right)\right]-\Pi_{\mathcal{G}}\left(H I_{A^{c}}\right)\right\}
\end{aligned}
$$

where the first equation holds by the definition of $\Pi^{*}$. The third equation holds because of Lemma A.4 for $r=2$. (A.4) yields that

$$
\begin{aligned}
& \Pi_{\mathcal{G}}^{*}\left(\frac{d \mathbb{Q}_{\mathcal{G}}}{d \mathbb{P}_{\mathcal{G}}}\left(Z_{1} I_{A}+Z_{2} I_{A^{c}}\right)\right) \leq \operatorname{ess}_{\sup _{H \in L^{\infty}}(\mathcal{F})}\left\{\mathbb{E}_{\mathcal{G}}\left[\frac{d \mathbb{Q}_{\mathcal{G}}}{d \mathbb{P}_{\mathcal{G}}} Z_{1} H\right]-\Pi_{\mathcal{G}}(H)\right\} \\
& +\operatorname{ess}_{\sup } \operatorname{seL}^{\infty}(\mathcal{F})\left\{\mathbb{E}_{\mathcal{G}}\left[\frac{d \mathbb{Q}_{\mathcal{G}}}{d \mathbb{P}_{\mathcal{G}}} Z_{2} H\right]-\Pi_{\mathcal{G}}(H)\right\} \\
& =\Pi_{\mathcal{G}}^{*}\left(\frac{d \mathbb{Q}_{\mathcal{G}}}{d \mathbb{P}_{\mathcal{G}}} Z_{1}\right)+\Pi_{\mathcal{G}}^{*}\left(\frac{d \mathbb{Q}_{\mathcal{G}}}{d \mathbb{P}_{\mathcal{G}}} Z_{2}\right)=0+0=0,
\end{aligned}
$$


where in the last line we have used that $\Pi_{\mathcal{G}}^{*}\left(\frac{d \mathbb{Q}_{\mathcal{G}}}{d \mathbb{P}_{\mathcal{G}}} Z_{i}\right)=0$. The reason for this is that $\Pi^{*}$ is zero on $M$ and infinity else. Therefore, the fact that $Z_{i} \in M^{\prime}$ implies by (3.4) that $\frac{d \mathbb{Q}_{\mathcal{G}}}{d \mathbb{P}_{\mathcal{G}}} Z_{i} \in \frac{d \mathbb{Q}_{\mathcal{G}}}{d \mathbb{P}_{\mathcal{G}}} M^{\prime}=M$ for $i=1,2$.

Since $\Pi_{\mathcal{G}}^{*}$ is only takes the values zero and infinity we must have that $\Pi_{\mathcal{G}}^{*}\left(\frac{d \mathbb{Q}_{\mathcal{G}}}{d \mathbb{P}_{\mathcal{G}}}\left(Z_{1} I_{A}+\right.\right.$ $\left.\left.Z_{2} I_{A^{c}}\right)\right)=0$. Thus, we can conclude that indeed $\frac{d \mathbb{Q}_{\mathcal{G}}}{d \mathbb{P}_{\mathcal{G}}}\left(Z_{1} I_{A}+Z_{2} I_{A^{c}}\right) \in M$. Therefore,

$$
Z_{1} I_{A}+Z_{2} I_{A^{c}} \in\left(\frac{d \mathbb{Q}_{\mathcal{G}}}{d \mathbb{P}_{\mathcal{G}}}\right)^{-1} M=M^{\prime}
$$

Proof of Theorem 3.10. (ii) $\Rightarrow$ (i): It is

$$
\Pi_{\mathcal{G}}\left(H^{S}+H\right)=\mathbb{E}^{\mathbb{Q}_{\mathcal{G}}}\left[\Pi_{\mathcal{F} S}\left(H^{S}+H\right)\right]=\mathbb{E}^{\mathbb{Q}_{\mathcal{G}}}\left[H^{S}\right]+\mathbb{E}^{\mathbb{Q}_{\mathcal{G}}}\left[\Pi_{\mathcal{F}^{S}}(H)\right]=\mathbb{E}^{\mathbb{Q}_{\mathcal{G}}}\left[H^{S}\right]+\Pi_{\mathcal{G}}(H),
$$

where we have used $\mathcal{F}^{S}$-conditional cash invariance in the second equation. This shows marketconsistency. Moreover,

$$
\begin{aligned}
\Pi_{\mathcal{G}}\left(I_{A} H_{1}+I_{A^{c}} H_{2}\right) & =\mathbb{E}^{\mathbb{Q}_{\mathcal{G}}}\left[\Pi_{\mathcal{F} S}\left(I_{A} H_{1}+I_{A^{c}} H_{2}\right)\right] \\
& =\mathbb{E}^{\mathbb{Q}_{\mathcal{G}}}\left[I_{A} \Pi_{\mathcal{F} S}\left(H_{1}\right)+I_{A^{c}} \Pi_{\mathcal{F}^{S}}\left(H_{2}\right)\right] \\
& =\mathbb{E}^{\mathbb{Q}_{\mathcal{G}}}\left[\Pi_{\mathcal{F}^{S}}\left(I_{A} H_{1}\right)+\Pi_{\mathcal{F}^{S}}\left(I_{A^{c}} H_{2}\right)\right]=\Pi_{\mathcal{G}}\left(I_{A} H_{1}\right)+\Pi_{\mathcal{G}}\left(I_{A^{c}} H_{2}\right),
\end{aligned}
$$

where we used the $\mathcal{F}^{S}$-local property in the second and Lemma A.5 in the third equation. that

(i) $\Rightarrow$ (ii): By Proposition 3.3 (iii) $\Rightarrow$ (ii) and positive homogeneity market-consistency imply

$$
\begin{aligned}
\Pi_{\mathcal{G}}(H) & =\operatorname{ess}_{\sup } \operatorname{siM}_{Z} \mathbb{E}_{\mathcal{G}}\left[\frac{d \mathbb{Q}_{\mathcal{G}}}{d \mathbb{P}_{\mathcal{G}}} Z H\right] \\
& =\operatorname{ess}_{\sup _{Z \in M^{\prime}}} \mathbb{E}_{\mathcal{G}}\left[\mathbb{E}_{\mathcal{F} S}\left[\frac{d \mathbb{Q}_{\mathcal{G}}}{d \mathbb{P}_{\mathcal{G}}} Z H\right]\right]=\operatorname{ess} \sup _{Z \in M^{\prime}} \mathbb{E}_{\mathcal{G}}\left[\frac{d \mathbb{Q}_{\mathcal{G}}}{d \mathbb{P}_{\mathcal{G}}} \mathbb{E}_{\mathcal{F} S}[Z H]\right],
\end{aligned}
$$

where we used in the third equation that $\frac{d \mathbb{Q}_{\mathcal{G}}}{d \mathbb{P}_{\mathcal{G}}}$ is $\mathcal{F}^{S}$-measurable. Define

$$
\Pi_{\mathcal{F}^{S}}(H):=\operatorname{ess}_{\sup } \operatorname{seM}^{\prime} \mathbb{E}_{\mathcal{F}^{S}}[Z H] .
$$

Clearly, $\Pi_{\mathcal{F}^{S}}$ is normal, $\mathcal{F}^{S}$-convex, $\mathcal{F}^{S}$-cash invariant and $\mathcal{F}^{S}$-positively homogeneous. The $\mathcal{F}^{S}$-local property is satisfied because for $A \in \mathcal{F}^{S}$ we have

$$
\begin{aligned}
& \Pi_{\mathcal{F}^{S}}\left(I_{A} H_{1}+I_{A^{c}} H_{2}\right)=\operatorname{ess}_{\sup _{Z \in M^{\prime}}} \mathbb{E}_{\mathcal{F}^{S}}\left[Z I_{A} H_{1}+Z I_{A^{c}} H_{2}\right] \\
& =\operatorname{ess} \sup _{Z \in M^{\prime}} \mathbb{E}_{\mathcal{F S}}\left[Z I_{A} H_{1}\right]+\mathbb{E}_{\mathcal{F} S}\left[Z I_{A^{c}} H_{2}\right] \\
& =\operatorname{ess} \sup _{Z_{1} \in M^{\prime}, Z_{2} \in M^{\prime}} \mathbb{E}_{\mathcal{F}^{S}}\left[Z_{1} I_{A} H_{1}\right]+\mathbb{E}_{\mathcal{F}^{S}}\left[Z_{2} I_{A^{c}} H_{2}\right] \\
& =\operatorname{ess}_{\sup _{Z_{1} \in M^{\prime}}} \mathbb{E}_{\mathcal{F} S}\left[Z_{1} I_{A} H_{1}\right]+\operatorname{ess} \sup _{Z_{2} \in M^{\prime}} \mathbb{E}_{\mathcal{F S}}\left[Z_{2} I_{A^{c}} H_{2}\right] \\
& =I_{A} \operatorname{ess} \sup _{Z_{1} \in M^{\prime}} \mathbb{E}_{\mathcal{F} S}\left[Z_{1} H_{1}\right]+I_{A^{c}} \operatorname{ess}_{\sup _{Z_{2} \in M^{\prime}}} \mathbb{E}_{\mathcal{F} S}\left[Z_{2} H_{2}\right] \\
& =I_{A} \Pi_{\mathcal{F} S}\left(H_{1}\right)+I_{A^{c}} \Pi_{\mathcal{F} S}\left(H_{2}\right),
\end{aligned}
$$

where we used in the third equation that Lemma A.6 implies that $M^{\prime}=\left\{Z_{1} I_{A}+Z_{2} I_{A^{c}} \mid Z_{1} \in\right.$ $\left.M^{\prime}, Z_{2} \in M^{\prime}\right\}$. Hence, indeed $\Pi_{\mathcal{F}^{S}}$ is an $\mathcal{F}^{S}$-conditional evaluation. Finally, let us prove that

$$
\Pi_{\mathcal{G}}(H)=\mathbb{E}^{\mathbb{Q}_{\mathcal{G}}}\left[\Pi_{\mathcal{F}^{S}}(H)\right] .
$$


Notice that if we could show that

$$
\operatorname{ess} \sup _{Z \in M^{\prime}} \mathbb{E}_{\mathcal{G}}\left[\frac{d \mathbb{Q}_{\mathcal{G}}}{d \mathbb{P}_{\mathcal{G}}} \mathbb{E}_{\mathcal{F}^{S}}[Z H]\right]=\mathbb{E}_{\mathcal{G}}\left[\frac{d \mathbb{Q}_{\mathcal{G}}}{d \mathbb{P}_{\mathcal{G}}} \operatorname{ess} \sup _{Z \in M^{\prime}} \mathbb{E}_{\mathcal{F}^{S}}[Z H]\right]
$$

then we are done, since the left-hand side of (A.6) is equal to $\Pi_{\mathcal{G}}(H)$ by (A.5), while the righthand-side is equal to $\mathbb{E}^{\mathbb{Q}_{\mathcal{G}}}\left[\Pi_{\mathcal{F} S}(H)\right]$ by the definition of $\Pi_{\mathcal{F} S}$. So let us show (A.6). Clearly,

$$
\operatorname{ess}_{\sup _{Z \in M^{\prime}}} \mathbb{E}_{\mathcal{G}}\left[\frac{d \mathbb{Q}_{\mathcal{G}}}{d \mathbb{P}_{\mathcal{G}}} \mathbb{E}_{\mathcal{F}^{S}}[Z H]\right] \leq \mathbb{E}_{\mathcal{G}}\left[\frac{d \mathbb{Q}_{\mathcal{G}}}{d \mathbb{P}_{\mathcal{G}}} \operatorname{ess}_{\sup _{Z \in M^{\prime}}} \mathbb{E}_{\mathcal{F}^{S}}[Z H]\right]
$$

Let us prove ' $\geq$ '. It is well known, see also Theorem A.1 and Lemma A.3, that the concatenation property implies that there exists a sequence $Z_{n} \in M^{\prime}$ with $\mathbb{E}_{\mathcal{F}^{S}}\left[Z_{1} H\right] \leq \mathbb{E}_{\mathcal{F}^{S}}\left[Z_{2} H\right] \leq \ldots$ such that $\lim _{n} \mathbb{E}_{\mathcal{F} S}\left[Z_{n} H\right]=\operatorname{ess}_{\sup _{Z \in M^{\prime}}} \mathbb{E}_{\mathcal{F} S}[Z H]$. Therefore, by the monotone convergence theorem

$$
\begin{aligned}
\mathbb{E}_{\mathcal{G}}\left[\frac{d \mathbb{Q}_{\mathcal{G}}}{d \mathbb{P}_{\mathcal{G}}} \operatorname{ess}_{\sup _{Z \in M^{\prime}}} \mathbb{E}_{\mathcal{F} S}[Z H]\right] & =\lim _{n} \mathbb{E}_{\mathcal{G}}\left[\frac{d \mathbb{Q}_{\mathcal{G}}}{d \mathbb{P}_{\mathcal{G}}} \mathbb{E}_{\mathcal{F} S}\left[Z_{n} H\right]\right] \\
& \leq \operatorname{ess}_{\sup _{Z \in M^{\prime}}} \mathbb{E}_{\mathcal{G}}\left[\frac{d \mathbb{Q}_{\mathcal{G}}}{d \mathbb{P}_{\mathcal{G}}} \mathbb{E}_{\mathcal{F} S}[Z H]\right]
\end{aligned}
$$

This shows (A.6). This proves Theorem 3.10.

For the proof of Theorem 3.11 we will need the following Corollary of Lemma A.4:

Corollary A.7 Suppose that $\Pi_{\mathcal{G}}$ is continuous, market-consistent and satisfies the local property. Then for disjoint sets $C_{1}, C_{2}, \ldots \in \mathcal{F}^{S}$ and a payoff $H \in L^{\infty}(\mathcal{F})$ we have

$$
\Pi_{\mathcal{G}}\left(H \sum_{i=1}^{\infty} I_{C_{i}}\right)=\sum_{i=1}^{\infty} \Pi_{\mathcal{G}}\left(H I_{C_{i}}\right)
$$

In particular, if $\Pi_{\mathcal{G}}$ is additionally assumed to be monotone (or p-norm bounded) then for every $H \in L^{\infty}(\mathcal{F})$ the mapping $C \rightarrow \Pi_{\mathcal{G}}\left(H I_{C}\right)$ is a real-valued, (signed) measure on $\mathcal{F}^{S}$.

Proof of Theorem 3.11. (ii) $\Rightarrow(\mathrm{i})$ : Continuity, convexity, and monotonicity in the case that $\Pi_{\mathcal{F} S}$ is monotone, are straight-forward. The other properties are seen analogously as in the proof of Theorem 3.10.

(i) $\Rightarrow$ (ii): By assumption $\mathcal{F}^{S}=\sigma\left(A_{1}, A_{2}, \ldots\right)$ and we will assume without loss of generality that $A_{i} \neq A_{j}$ if $i \neq j$. For $n \in \mathbb{N}$ we define the finite filtration $\mathcal{F}^{n}$ as the smallest $\sigma$-algebra containing the the events $A_{1}, \ldots, A_{n}$. Now let us define the partitions corresponding to $\mathcal{F}^{n}$ recursively in a standard way. For $n=1, \mathcal{F}^{1}$ is generated by the partition given by $B_{1}^{1}:=A_{1}$, $B_{2}^{1}:=A_{1}^{c}$. Moreover, $\mathcal{F}^{n+1}$ is generated by the partition

$$
B_{i}^{n+1}:=B_{i}^{n} \cap A_{n+1} \text { and } B_{2^{n}+i}^{n+1}:=B_{i}^{n} \cap A_{n+1}^{c} \quad i=1, \ldots, 2^{n} .
$$

Of course, $\mathcal{F}^{1} \subset \mathcal{F}^{2} \subset \ldots \subset \mathcal{F}^{S}$.

Set $q_{k}^{n}=\mathbb{Q}_{\mathcal{G}}\left(B_{k}^{n}\right)$. Note that $q_{k}^{n}$ are $\mathcal{G}$-measurable random variables summing up to one for a.s. all fixed $\omega$. Define

$$
\Pi_{\mathcal{F}^{n}}(H):=\sum_{k=1}^{2^{n}} \frac{I_{B_{k}^{n}}}{q_{k}^{n}} \Pi_{\mathcal{G}}\left(H I_{B_{k}^{n}}\right)
$$


where we we set $0 / 0=0$. Note that if $q_{k}^{n}=\mathbb{Q}_{\mathcal{G}}\left[B_{k}^{n}\right]=0$ on a non-zero set $C \in \mathcal{G}$, then $\mathbb{P}_{\mathcal{G}}\left[B_{k}^{n}\right]=0$ on $C$ as well (as $\mathbb{Q}_{\mathcal{G}}$ is equivalent to $\mathbb{P}_{\mathcal{G}}$ ) and therefore, $\Pi_{\mathcal{G}}\left(H I_{B_{k}^{n}}\right)=0$ on $C$, too. In particular, $\Pi_{\mathcal{F} n}$ is well defined.

Next, every set $A \in \mathcal{F}^{n}$ can be written as $A=B_{k_{1}}^{n} \cup \ldots \cup B_{k_{r}}^{n}$, for a $r \in\left\{1, \ldots, 2^{n}\right\}$ and $1 \leq k_{1}<k_{2}<\ldots<k_{r} \leq 2^{n}$. As $\Pi_{\mathcal{G}}(0)=0$, it is straight-forward to check using Definition (A.7) that

$$
\Pi_{\mathcal{F}^{n}}\left(I_{A} H\right)=I_{A} \Pi_{\mathcal{F}^{n}}(H) .
$$

Furthermore, by Lemma A.4 for every $H \in L^{\infty}(\mathcal{F})$

$$
\mathbb{E}^{\mathbb{Q}_{\mathcal{G}}}\left[\Pi_{\mathcal{F}^{n}}(H)\right]=\sum_{k=1}^{2^{n}} \frac{\mathbb{E}^{\mathbb{Q}_{\mathcal{G}}}\left[I_{B_{k}^{n}}\right]}{q_{k}^{n}} \Pi_{\mathcal{G}}\left(H I_{B_{k}^{n}}\right)=\Pi_{\mathcal{G}}(H) .
$$

We will need the following lemma which is a version of Proposition A.12 in Föllmer and Schied (2004).

Lemma A.8 Suppose that $\hat{\mathbb{P}}$ and $\overline{\mathbb{P}}$ share the same zero sets, and that $\mathcal{F}_{0} \subset \mathcal{F}$. Then for any bounded $\mathcal{F}$-measurable $H$

$$
\hat{\mathbb{E}}_{\mathcal{F}_{0}}[H]=\frac{1}{\overline{\mathbb{E}}_{\mathcal{F}_{0}}[d \hat{\mathbb{P}} / d \overline{\mathbb{P}}]} \overline{\mathbb{E}}_{\mathcal{F}_{0}}\left[\frac{d \hat{\mathbb{P}}}{d \overline{\mathbb{P}}} H\right] \quad \overline{\mathbb{P}} \text { and } \hat{\mathbb{P}} \text { a.s. }
$$

Next we will show the following Lemma:

Lemma A.9 Under our assumptions, for every $H \in L^{\infty}(\mathcal{F})$ the process $M_{n}=\mathbb{E}^{\mathbb{Q}_{\mathcal{G}}}\left[\Pi_{\mathcal{F}^{n}}(H)\right]$ is a uniformly integrable martingale.

Proof. It may be seen from standard arguments that $M_{n}=\Pi_{\mathcal{F}^{n}}(H)$ is a martingale. Let us see that $M_{n}$ is uniformly integrable under the measure $\mathbb{Q}_{\mathcal{G}}$. First of all note that in the case that $\Pi_{\mathcal{G}}$ is monotone we have that

$$
\Pi_{\mathcal{F}^{n}}(H) \leq \sum_{k=1}^{2^{n}} \frac{I_{B_{k}^{n}}}{q_{k}^{n}} \Pi_{\mathcal{G}}\left(\|H\|_{\infty} I_{B_{k}^{n}}\right)=\sum_{k=1}^{2^{n}} \frac{I_{B_{k}^{n}}}{q_{k}^{n}}\|H\|_{\infty} q_{k}^{n}=\|H\|_{\infty},
$$

where we used market-consistency in the first equation. Similarly, it is seen that $\Pi_{\mathcal{F}^{n}}(H) \geq$ $-\|H\|_{\infty}$. Hence, if $\Pi_{\mathcal{F}^{n}}$ is monotone then for fixed $H, \Pi_{\mathcal{F}^{n}}(H)$ is bounded uniformly. In particular, $M_{n}$ is uniformly integrable.

In the case that $\Pi_{\mathcal{G}}$ is $p$-norm bounded notice that,

$$
\begin{aligned}
\left|\Pi_{\mathcal{F}^{n}}(H)\right| & \leq \lambda\left|\sum_{k=1}^{2^{n}} \frac{I_{B_{k}^{n}}}{q_{k}^{n}} \int\left(|H|+|H|^{p}\right) I_{B_{k}^{n}} d \overline{\mathbb{P}}_{\mathcal{G}}\right| \\
& \leq \lambda\left(|| H\left\|_{\infty}+\right\| H \|_{\infty}^{p}\right)\left|\sum_{k=1}^{2^{n}} I_{B_{k}^{n}} \frac{\overline{\mathbb{P}}_{\mathcal{G}}\left(B_{k}^{n}\right)}{\mathbb{Q}_{\mathcal{G}}\left(B_{k}^{n}\right)}\right|=\lambda\left(\|H\|_{\infty}+\|H\|_{\infty}^{p}\right) \mathbb{E}^{\mathbb{Q}_{\mathcal{G}}}\left[\frac{d \overline{\mathbb{P}}_{\mathcal{G}}}{d \mathbb{Q}_{\mathcal{G}}} \mid \mathcal{F}^{n}\right] .
\end{aligned}
$$

Since the last term is uniformly integrable, $\Pi_{\mathcal{F}^{n}}(H)$ is uniformly integrable as well. 
Hence, if $\Pi_{\mathcal{G}}$ is monotone or $p$-norm bounded we may conclude by the martingale convergence theorem that $M_{n}=\Pi_{\mathcal{F} n}(H)$ converges a.s. and in $L^{1}\left(\mathbb{Q}_{\mathcal{G}}\right)$ to a random variable $M_{\infty}$. Set

$$
\Pi_{\mathcal{F} S}(H):=M_{\infty}=\lim _{n} \Pi_{\mathcal{F}^{n}}(H) .
$$

Now $\Pi_{\mathcal{G}}(H)=\mathbb{E}^{\mathbb{Q}_{\mathcal{G}}}\left[\Pi_{\mathcal{F} S}(H)\right]$ will follow from the following lemma:

Lemma A.10 For all $A \in \mathcal{F}^{S}$ and $H \in L^{\infty}(\mathcal{F}), \Pi_{\mathcal{F}^{S}}(H)$ satisfies the characteristic equation:

$$
\Pi_{\mathcal{G}}\left(I_{A} H\right)=\mathbb{E}^{\mathbb{Q}_{\mathcal{G}}}\left[I_{A} \Pi_{\mathcal{F} S}(H)\right] .
$$

In particular, $\Pi_{\mathcal{G}}(H)=\mathbb{E}^{\mathbb{Q}_{\mathcal{G}}}\left[\Pi_{\mathcal{F} S}(H)\right]$. Furthermore, for every $H \in L^{\infty}\left(\mathcal{F}^{S}\right), \Pi_{\mathcal{F} S}(H)$ is the unique a.s. $\mathbb{Q}_{\mathcal{G}}$-integrable random variable satisfying (A.9).

Proof. Notice that for every $A \in \mathcal{F}^{n_{0}} \subset \mathcal{F}^{n_{0}+1} \subset \ldots$ by (A.8)

$$
\Pi_{\mathcal{G}}\left(I_{A} H\right)=\lim _{n} \mathbb{E}^{\mathbb{Q}_{\mathcal{G}}}\left[\Pi_{\mathcal{F}^{n}}\left(I_{A} H\right)\right]=\lim _{n} \mathbb{E}^{\mathbb{Q}_{\mathcal{G}}}\left[I_{A} \Pi_{\mathcal{F}^{n}}(H)\right]=\mathbb{E}^{\mathbb{Q}_{\mathcal{G}}}\left[I_{A} \Pi_{\mathcal{F} S}(H)\right],
$$

where we have used in the third equality that $\Pi_{\mathcal{F} n}(H)$ converges to $\Pi_{\mathcal{F} S}(H)$ in $L^{1}\left(\mathbb{Q}_{\mathcal{G}}\right)$. Now by Corollary A.7 the left hand-side and the right hand-side are signed measures (measuring sets $A \in \mathcal{F}^{S}$ ). As by (A.10) they both agree on $\bigcup_{n} \mathcal{F}^{n}$, which is closed under intersection and generates $\mathcal{F}^{S}$, they must agree on the entire filtration $\mathcal{F}^{S}$. Uniqueness follows from equation (A.9) using standard arguments.

Now all what is left to prove is the following lemma:

Lemma A.11 $\Pi_{\mathcal{F} S}$ is a continuous, $\mathcal{F}^{S}$-conditional evaluation which is monotone if $\Pi_{\mathcal{G}}$ is monotone.

Proof. Clearly for every $n \Pi_{\mathcal{F}^{n}}$ is $\mathcal{F}^{n}$ - and hence also $\mathcal{F}^{S}$-measurable. This entails that its limit, $\Pi_{\mathcal{F}^{S}}$, is $\mathcal{F}^{S}$-measurable. Furthermore, by construction, $\Pi_{\mathcal{F}^{S}}$ is normalized as $\Pi_{\mathcal{F}^{n}}$ are. If $\Pi_{\mathcal{G}}$ is monotone then the $\Pi_{\mathcal{F}^{n}}$ are monotone as well which implies that $\Pi_{\mathcal{F}^{S}}$ is monotone.

Next, let us check that $\Pi_{\mathcal{F} S}$ satisfies the $\mathcal{F}^{S}$-local property. It is necessary and sufficient that for every $A \in \mathcal{F}^{S}$ and $H \in L^{\infty}(\mathcal{F})$

$$
\Pi_{\mathcal{F} S}\left(H I_{A}\right)=I_{A} \Pi_{\mathcal{F} S}(H) .
$$

We will prove the equality by showing that the right-hand side satisfies, the characteristic equation (A.9) for the left hand-side. So let $A^{\prime} \in \mathcal{F}^{S}$. It is

$$
\Pi_{\mathcal{G}}\left(I_{A^{\prime}}\left(I_{A} H\right)\right)=\Pi_{\mathcal{G}}\left(I_{A^{\prime} \cap A} H\right)=\mathbb{E}^{\mathbb{Q}_{\mathcal{G}}}\left[I_{A^{\prime} \cap A} \Pi_{\mathcal{F}^{S}}(H)\right]=\mathbb{E}^{\mathbb{Q}_{\mathcal{G}}}\left[I_{A^{\prime}}\left(I_{A} \Pi_{\mathcal{F} S}(H)\right)\right],
$$

where we have used (A.9) in the second equation. This shows that $I_{A} \Pi_{\mathcal{F}^{S}}(H)$ satisfies the characteristic equation of $I_{A} H$ and hence by the uniqueness stated in Lemma A.10 indeed $\Pi_{\mathcal{F} S}\left(H I_{A}\right)=I_{A} \Pi_{\mathcal{F} S}(H)$.

To see $\mathcal{F}^{S}$-cash invariance of $\Pi_{\mathcal{F} S}$ assume that for a $m \in L^{\infty}\left(\mathcal{F}^{S}\right)$ and $H \in L^{\infty}(\mathcal{F})$ we have that the set $A=\left\{\Pi_{\mathcal{F} S}(H+m) \stackrel{(<)}{>} \Pi_{\mathcal{F} S}(H)+m\right\}$ has positive measure under $\mathbb{P}_{\mathcal{G}}$. Then $A$ has also positive measure under $\mathbb{Q}_{\mathcal{G}}$ and

$$
\begin{aligned}
\Pi_{\mathcal{G}}\left(H I_{A}+m I_{A}\right) & =\mathbb{E}^{\mathbb{Q}_{\mathcal{G}}}\left[\Pi_{\mathcal{F} S}\left((H+m) I_{A}\right)\right]=\mathbb{E}^{\mathbb{Q}_{\mathcal{G}}}\left[\Pi_{\mathcal{F}^{S}}(H+m) I_{A}\right] \\
& \stackrel{(<)}{>} \mathbb{E}^{\mathbb{Q}_{\mathcal{G}}}\left[\left(\Pi_{\mathcal{F} S}(H)+m\right) I_{A}\right] \\
& =\mathbb{E}^{\mathbb{Q}_{\mathcal{G}}}\left[\Pi_{\mathcal{F} S}\left(H I_{A}\right)\right]+\mathbb{E}^{\mathbb{Q}_{\mathcal{G}}}\left[m I_{A}\right]=\Pi_{\mathcal{G}}\left(H I_{A}\right)+\mathbb{E}^{\mathbb{Q}_{\mathcal{G}}}\left[m I_{A}\right]
\end{aligned}
$$


where we have used in the second equation the $\mathcal{F}^{S}$-local property of $\Pi_{\mathcal{F}^{S}}$, which we have proved above. This is a contradiction to the market-consistency of $\Pi_{\mathcal{G}}$. Thus, indeed $\Pi_{\mathcal{F}^{S}}$ is $\mathcal{F}^{S}$-cash invariant.

Next, for $H \in L^{\infty}(\mathcal{F})$, and $\lambda \in \mathbb{R}$ with $0 \leq \lambda \leq 1$

$$
\begin{aligned}
\Pi_{\mathcal{F} n}\left(\lambda H_{1}+(1-\lambda) H_{2}\right) & =\sum_{k=1}^{2^{n}} \frac{I_{B_{k}^{n}}}{q_{k}^{n}} \Pi_{\mathcal{G}}\left(\lambda I_{B_{k}^{n}} H_{1}+(1-\lambda) I_{B_{k}^{n}} H_{2}\right) \\
& \leq \sum_{k=1}^{2^{n}} \frac{I_{B_{k}^{n}}}{q_{k}^{n}}\left(\lambda \Pi_{\mathcal{G}}\left(I_{B_{k}^{n}} H_{1}\right)+(1-\lambda) \Pi_{\mathcal{G}}\left(I_{B_{k}^{n}} H_{2}\right)\right) \\
& \left.=\lambda \Pi_{\mathcal{F}^{n}}\left(H_{1}\right)+(1-\lambda) \Pi_{\mathcal{F}^{n}} H_{2}\right),
\end{aligned}
$$

where we have used the convexity of $\Pi_{\mathcal{G}}$ in the inequality. In particular, $\Pi_{\mathcal{F} S}$ is convex as limit of convex functionals. (Note that 'convexity' is weaker than ' $\mathcal{F}^{S}$-convexity' since for 'convexity' $\lambda \in[0,1]$ is assumed to be deterministic.) Before we move on to show the $\mathcal{F}^{S}$-convexity of $\Pi_{\mathcal{F} S}$ let us prove continuity.

In the case that $\Pi_{\mathcal{G}}$ is monotone, $\Pi_{\mathcal{F}^{S}}$ is monotone as well and the continuity of $\Pi_{\mathcal{F}^{S}}$ follows from the characteristic equation using standard arguments.

On the other hand if $\Pi_{\mathcal{G}}$ is $p$-norm bounded we get for $H \in L^{\infty}(\mathcal{F})$

$$
\begin{aligned}
\left|\Pi_{\mathcal{F}^{n}}(H)\right| & \leq \lambda\left|\sum_{k=1}^{2^{n}} \frac{I_{B_{k}^{n}}}{q_{k}^{n}} \int\left(|H|+|H|^{p}\right) I_{B_{k}^{n}} d \overline{\mathbb{P}}_{\mathcal{G}}\right| \\
& =\lambda\left|\sum_{k=1}^{2^{n}} \frac{I_{B_{k}^{n}}}{q_{k}^{n}} \int\left(|H|+|H|^{p}\right) I_{B_{k}^{n}} \frac{d \overline{\mathbb{P}}_{\mathcal{G}}}{d \mathbb{Q}_{\mathcal{G}}} d \mathbb{Q}_{\mathcal{G}}\right|=\lambda \mathbb{E}^{\mathbb{Q}_{\mathcal{G}}}\left[\left(|H|+|H|^{p}\right) \frac{d \overline{\mathbb{P}}_{\mathcal{G}}}{d \mathbb{Q}_{\mathcal{G}}} \mid \mathcal{F}^{n}\right] .
\end{aligned}
$$

Therefore,

$$
\begin{aligned}
\left|\Pi_{\mathcal{F} S}(H)\right| & =\lim _{n}\left|\Pi_{\mathcal{F}^{n}}(H)\right| \\
& \leq \lambda \mathbb{E}^{\mathbb{Q}_{\mathcal{G}}}\left[\left(|H|+|H|^{p}\right) \frac{d \overline{\mathbb{P}}_{\mathcal{G}}}{d \mathbb{Q}_{\mathcal{G}}} \mid \mathcal{F}^{S}\right] \\
& =\lambda \mathbb{E}^{\mathbb{Q}_{\mathcal{G}}}\left[\frac{d \overline{\mathbb{P}}_{\mathcal{G}}}{d \mathbb{Q}_{\mathcal{G}}} \mid \mathcal{F}^{S}\right] \frac{\mathbb{E}^{\mathbb{Q}_{\mathcal{G}}}\left[\left(|H|+|H|^{p}\right) \frac{d \overline{\mathbb{P}}_{\mathcal{G}}}{d \mathbb{Q}_{\mathcal{G}}} \mid \mathcal{F}^{S}\right]}{\mathbb{E}^{\mathbb{Q}_{\mathcal{G}}}\left[\frac{d \overline{\mathbb{P}}_{\mathcal{G}}}{d \mathbb{Q}_{\mathcal{G}}} \mid \mathcal{F}^{S}\right]} \\
& =\lambda \mathbb{E}^{\mathbb{Q}_{\mathcal{G}}}\left[\frac{d \overline{\mathbb{P}}_{\mathcal{G}}}{d \mathbb{Q}_{\mathcal{G}}} \mid \mathcal{F}^{S}\right] \int\left(|H|+|H|^{p}\right) d \overline{\mathbb{P}}_{\mathcal{F}^{S} .}
\end{aligned}
$$

The third equation holds by Lemma A.8. Now by $\left(\right.$ A.12) we can extend $\Pi_{\mathcal{F} S}$ to $L^{p}(\Omega, \mathcal{F}, \overline{\mathbb{P}})$ by setting

$$
\tilde{\Pi}_{\mathcal{F}^{S}}(H)=\limsup _{N \rightarrow \infty} \limsup _{m \rightarrow \infty} \Pi_{\mathcal{F}^{S}}(-m \vee H \wedge N) .
$$

Note that we have that $\tilde{\Pi}_{\mathcal{F} S}$ is convex (as the limsup of convex functionals), and agrees with $\Pi_{\mathcal{F} S}$ on $L^{\infty}(\mathcal{F})$. Define $L^{p}\left(\Omega, \mathcal{F}, \overline{\mathbb{P}}_{\mathcal{F} S}\right)$ as all random variables $H$ such that $\left.\left(\int\left|H\left(\omega^{\prime}\right)\right|^{p}\left(\omega^{\prime}\right) \overline{\mathbb{P}}_{\mathcal{F} S}\right)\left(d \omega^{\prime}\right)\right)^{1 / p}:=$ $\left(\mathbb{E}_{\mathcal{F} S}\left[|H|^{p} \bar{\xi}\right]\right)^{1 / p}<\infty$, where $\bar{\xi}$ is the conditional density corresponding to $\overline{\mathbb{P}}_{\mathcal{F} S}$. By (A.12), for 
every $H$ we have that $\tilde{\Pi}_{\mathcal{F}^{S}}(H)$ is real-valued and uniformly and bounded in any $L^{p}\left(\Omega, \mathcal{F}, \overline{\mathbb{P}}_{\mathcal{F} S}\right)$ environment around $H$. It follows then from standard arguments for convex functionals, see for instance Theorem 2.2.9 in Zălinescu (2002), that convergence of $H_{n}$ to $H$ in $L^{p}\left(\Omega, \mathcal{F}, \overline{\mathbb{P}}_{\mathcal{F} S}\right)$ implies that $\tilde{\Pi}_{\mathcal{F} S}\left(H_{n}\right)$ converges to $\tilde{\Pi}_{\mathcal{F} S}(H)$. Since $\tilde{\Pi}_{\mathcal{F}^{S}}$ and $\Pi_{\mathcal{F}^{S}}$ agree on $L^{\infty}(\mathcal{F})$ we may concluded that indeed $\Pi_{\mathcal{F} S}$ is continuous with respect to bounded a.s. convergence.

Finally let us show that $\Pi_{\mathcal{F} S}$ is $\mathcal{F}^{S}$-convex. First of all, let $\lambda^{\mathcal{F}^{n}} \in L^{\infty}\left(\mathcal{F}^{n}\right)$ with $0 \leq$ $\lambda^{\mathcal{F}^{n}} \leq 1$. Then there exists disjoint sets $A_{1}^{n}, \ldots, A_{r}^{n} \in \mathcal{F}^{n}$ and constants $\lambda_{1}^{n}, \ldots, \lambda_{r}^{n} \in[0,1]$ with $\lambda^{\mathcal{F}^{n}}=\sum_{j=1}^{r} \lambda_{i}^{n} I_{A_{i}^{n}}$. By adding an additional set with an additional constant equal to zero if necessary, we may assume without loss of generality that $\Omega=\bigcup_{i=1}^{r} A_{i}^{n}$. By Lemma A.4 for sets $C_{1}, \ldots, C_{r} \in \mathcal{F}^{S}$

$$
\begin{aligned}
\Pi_{\mathcal{F}}\left(\sum_{i=1}^{r} I_{C_{i}} H_{i}\right) & =\sum_{k=1}^{2^{n}} \frac{I_{B_{k}^{n}}}{q_{k}^{n}}\left(\Pi_{\mathcal{G}}\left(\sum_{i=1}^{r} I_{B_{k}^{n}} I_{C_{i}} H_{i}\right)\right) \\
& =\sum_{i=1}^{r} \sum_{k=1}^{2^{n}} \frac{I_{B_{k}^{n}}}{q_{k}^{n}}\left(\Pi_{\mathcal{G}}\left(I_{B_{k}^{n}} I_{C_{i}} H_{i}\right)\right)=\sum_{i=1}^{r} \Pi_{\mathcal{F}^{n}}\left(I_{C_{i}} H_{i}\right) .
\end{aligned}
$$

Therefore,

$$
\begin{aligned}
\Pi_{\mathcal{F}^{n}}\left(\lambda^{\mathcal{F}^{n}} H_{1}+\left(1-\lambda^{\mathcal{F}^{n}}\right) H_{2}\right) & =\Pi_{\mathcal{F}^{n}}\left(\sum_{i=1}^{r} I_{A_{i}^{n}}\left(\lambda_{i}^{n} H_{1}+\left(1-\lambda_{i}^{n}\right) H_{2}\right)\right) \\
& =\sum_{i=1}^{r} \Pi_{\mathcal{F}^{n}}\left(\lambda_{i}^{n} I_{A_{i}^{n}} H_{1}+\left(1-\lambda_{i}^{n}\right) I_{A_{i}^{n}} H_{2}\right) \\
& \leq \sum_{i=1}^{r} \lambda_{i}^{n} \Pi_{\mathcal{F}^{n}}\left(I_{A_{i}^{n}} H_{1}\right)+\left(1-\lambda_{i}^{n}\right) \Pi_{\mathcal{F}^{n}}\left(I_{A_{i}^{n}} H_{2}\right) \\
& =\sum_{i=1}^{r} \lambda_{i}^{n} I_{A_{i}^{n}} \Pi_{\mathcal{F}^{n}}\left(H_{1}\right)+\sum_{i=1}^{r}\left(1-\lambda_{i}^{n}\right) I_{A_{i}^{n}} \Pi_{\mathcal{F}^{n}}\left(H_{2}\right) \\
& =\lambda^{\mathcal{F}^{n}} \Pi_{\mathcal{F}^{n}}\left(H_{1}\right)+\left(1-\lambda^{\mathcal{F}^{n}}\right) \Pi_{\mathcal{F}^{n}}\left(H_{2}\right),
\end{aligned}
$$

where we have used (A.13) in the second equation, (A.11) in the inequality, and the $\mathcal{F}^{n}$-local property for $\Pi_{\mathcal{F}^{n}}$, proved in (A.8), in the third equation. Hence, indeed $\Pi_{\mathcal{F}^{n}}$ is $\mathcal{F}^{n}$-convex.

Next, if $\lambda^{\mathcal{F}^{m}} \in L^{\infty}\left(\mathcal{F}^{m}\right)$ with $0 \leq \lambda^{\mathcal{F}^{m}} \leq 1$, and $m \in \mathbb{N}$ then clearly $\lambda^{\mathcal{F}^{m}} \in \mathcal{F}^{n}$ for every $n \geq m$. This entails

$$
\begin{aligned}
\Pi_{\mathcal{F} S}\left(\lambda^{\mathcal{F}^{m}} H_{1}+\left(1-\lambda^{\mathcal{F}^{m}}\right) H_{2}\right) & =\lim _{n} \Pi_{\mathcal{F}^{n}}\left(\lambda^{\mathcal{F}^{m}}\left(H_{1}+\left(1-\lambda^{\mathcal{F}^{m}}\right) H_{2}\right)\right. \\
& \leq \lim _{n} \lambda^{\mathcal{F}^{m}} \Pi_{\mathcal{F} n}\left(H_{1}\right)+\left(1-\lambda^{\mathcal{F}^{m}}\right) \Pi_{\mathcal{F} m}\left(H_{2}\right) \\
& =\lambda^{\mathcal{F}^{m}} \Pi_{\mathcal{F}^{S}}\left(H_{1}\right)+\left(1-\lambda^{\mathcal{F}^{m}}\right) \Pi_{\mathcal{F}^{m}}\left(H_{2}\right) .
\end{aligned}
$$

Finally, to see that $\Pi_{\mathcal{F} S}$ is $\mathcal{F}^{S}$-convex let $\lambda^{\mathcal{F}^{S}} \in L^{\infty}\left(\mathcal{F}^{S}\right)$ with $0 \leq \lambda^{\mathcal{F}^{S}} \leq 1$. Then also $0 \leq \mathbb{E}_{\mathcal{F}^{n}}\left[\lambda^{\mathcal{F}^{S}}\right] \leq 1$. Furthermore, by the martingale convergence theorem $\mathbb{E}_{\mathcal{F}^{n}}\left[\lambda^{S}\right]$ converges 
to $\mathbb{E}_{\mathcal{F} S}\left[\lambda^{S}\right]=\lambda^{S}$ a.s. Hence, by the continuity of $\Pi_{\mathcal{F} S}$ we obtain that

$$
\begin{aligned}
\Pi_{\mathcal{F} S}\left(\lambda^{\mathcal{F}^{S}} H_{1}+\left(1-\lambda^{\mathcal{F}^{S}}\right) H_{2}\right) & =\lim _{n} \Pi_{\mathcal{F}^{S}}\left(\mathbb{E}_{\mathcal{F}^{n}}\left[\lambda^{\mathcal{F}^{S}}\right] H_{1}+\left(1-\mathbb{E}_{\mathcal{F}^{n}}\left[\lambda^{\mathcal{F}^{S}}\right]\right) H_{2}\right) \\
& \leq \lim _{n} \mathbb{E}_{\mathcal{F}^{n}}\left[\lambda^{\mathcal{F}^{S}}\right] \Pi_{\mathcal{F}^{S}}\left(H_{1}\right)+\left(1-\mathbb{E}_{\mathcal{F}^{n}}\left[\lambda^{\mathcal{F}^{S}}\right]\right) \Pi_{\mathcal{F}^{S}}\left(H_{2}\right) \\
& =\lambda^{\mathcal{F}^{S}} \Pi_{\mathcal{F}^{S}}\left(H_{1}\right)+\left(1-\lambda^{\mathcal{F}^{S}}\right) \Pi_{\mathcal{F}^{S}}\left(H_{2}\right)
\end{aligned}
$$

where we have used (A.14) in the inequality. The lemma is proved.

Lemma A.10 and Lemma A.11 imply the theorem.

\section{A.3 Proofs of the results in Section 4}

For the proof of Theorem 4.3 we will need the following Lemma:

Lemma A.12 In the setting of Theorem 4.3, let $\tau$ be a stopping time such that $\sigma \leq \tau<\tau_{\sigma}$. Let $H$ be a bounded, $\mathcal{F}_{\tau}$-measurable payoff. Then $\Pi_{\sigma}(H)=\mathbb{E}_{\mathcal{F}_{\sigma}}^{\mathbb{Q}}[H]$.

Proof. As $\overline{\mathcal{F}}_{\tau_{i+1}-}^{Y}=\overline{\mathcal{F}}_{\tau_{i}}^{Y}$ for all $i, H$ is $\overline{\mathcal{F}}_{\tau}^{S} \vee \mathcal{F}_{\sigma^{-}}$measurable. Consequently, the lemma follows directly from the definition of market-consistency.

Proof of Theorem 4.3. As the jump times of $Y$ for the $i$-th jump, $\left(\tau_{i}\right)$, are predictable respectively, $\tau_{\sigma}$ has to be predictable as well. This is seen as follows: First of all note that by the predictability of $\tau_{i}$ there exists sequences of stopping times $\left(\tau_{i}^{n}\right)$ with $\tau_{i}^{n}<\tau_{i}$ and $\tau_{i}^{n} \uparrow \tau_{i}$ as $n \rightarrow \infty$. Define $\sigma^{n}:=\sum_{i=1}^{\infty} I_{\left\{\tau_{i}=\tau_{\sigma}\right\}}\left(\tau_{i}^{n} \vee \sigma\right)$. Let $\tau_{0}:=0$. Then $\left\{\tau_{i}=\tau_{\sigma}\right\}=\left\{\tau_{i-1} \leq \sigma<\right.$ $\left.\tau_{i}\right\} \in \mathcal{F}_{\sigma}$ for $i=1,2, \ldots$ (Since $\tau_{\sigma}$ is the first jump after time $\sigma$, we know at time $\sigma$, if we have observed $i-1$ jumps so far, so that the next jump will be the $i$-th one.) Therefore,

$$
\left\{\sigma^{n} \leq t\right\}=\bigcup_{i=1}^{\infty}\left(\left\{\tau_{i}^{n} \leq t\right\} \cap\{\sigma \leq t\} \cap\left\{\tau_{i}=\tau_{\sigma}\right\}\right) \in \mathcal{F}_{t} .
$$

Thus, $\sigma^{n}$ is indeed a stopping time. Furthermore, clearly $\sigma^{n}<\tau_{\sigma}$ and

$$
\sigma^{n} \uparrow \sum_{i=1}^{\infty} I_{\left\{\tau_{i}=\tau_{\sigma}\right\}}\left(\tau_{i} \vee \sigma\right)=\sum_{i=1}^{\infty} I_{\left\{\tau_{i}=\tau_{\sigma}\right\}}\left(\tau_{\sigma} \vee \sigma\right)=\sum_{i=1}^{\infty} I_{\left\{\tau_{i}=\tau_{\sigma}\right\}} \tau_{\sigma}=\tau_{\sigma},
$$

where we have used that $\sigma<\tau_{\sigma}$. Hence, $\tau_{\sigma}$ is indeed predictable.

Next, let $H \in L^{\infty}\left(\mathcal{F}_{\tau_{\sigma}}\right)$ and $A \in \mathcal{F}_{\sigma^{m}}$ for an $m \in \mathbb{N}$. By time-consistency and the local property of $\left(\Pi_{\sigma}\right)$ we get for all $n \geq m$

$$
\Pi_{\sigma}(H)=\Pi_{\sigma}\left(\Pi_{\sigma^{n}}\left(H I_{A}+H I_{A^{c}}\right)\right)=\Pi_{\sigma}\left(I_{A} \Pi_{\sigma^{n}}(H)+I_{A^{c}} \Pi_{\sigma^{n}}(H)\right) .
$$

Next observe that for $n \geq m, I_{A} \Pi_{\sigma^{n}}(H)+I_{A^{c}} \Pi_{\sigma^{n}}(H)$ is $\mathcal{F}_{\sigma^{n}}$-measurable. By Lemma A.12 this implies that

$$
\begin{aligned}
\Pi_{\sigma}\left(I_{A} \Pi_{\sigma^{n}}(H)+I_{A^{c}} \Pi_{\sigma^{n}}(H)\right) & =\mathbb{E}_{\mathcal{F}_{\sigma}}^{\mathbb{Q}}\left[I_{A} \Pi_{\sigma^{n}}(H)+I_{A^{c}} \Pi_{\sigma^{n}}(H)\right] \\
& =\mathbb{E}_{\mathcal{F}_{\sigma}}^{\mathbb{Q}}\left[I_{A} \Pi_{\sigma^{n}}(H)\right]+\mathbb{E}_{\mathcal{F}_{\sigma}}^{\mathbb{Q}}\left[I_{A^{c}} \Pi_{\sigma^{n}}(H)\right] \\
& =\mathbb{E}_{\mathcal{F}_{\sigma}}^{\mathbb{Q}}\left[\Pi_{\sigma^{n}}\left(I_{A} H\right)\right]+\mathbb{E}_{\mathcal{F}_{\sigma}}^{\mathbb{Q}}\left[\Pi_{\sigma^{n}}\left(I_{A^{c}} H\right)\right] \\
& =\Pi_{\sigma}\left(\Pi_{\sigma^{n}}\left(I_{A} H\right)\right)+\Pi_{\sigma}\left(\Pi_{\sigma^{n}}\left(I_{A^{c}} H\right)\right)=\Pi_{\sigma}\left(I_{A} H\right)+\Pi_{\sigma}\left(I_{A^{c}} H\right)
\end{aligned}
$$


where we used Lemma A.5 in the third and time-consistency in the last equation. Hence, for every $A \in \mathcal{F}_{\sigma^{m}}$ we have that

$$
\Pi_{\sigma}(H)=\Pi_{\sigma}\left(I_{A} H\right)+\Pi_{\sigma}\left(I_{A^{c}} H\right) .
$$

Since by assumption $S$ is continuous and $\overline{\mathcal{F}}_{\tau_{i+1}-}^{Y}=\overline{\mathcal{F}}_{\tau_{i}}^{Y}$ for all $i, \bigcup_{m} \mathcal{F}_{\sigma^{m}}$ is a generating system for $\mathcal{F}_{\tau_{\sigma}}^{S}$. By the continuity of $\Pi_{\sigma}$ this entails that (A.15) holds for all $A \in \mathcal{F}_{\tau_{\sigma}}^{S}$. Thus, indeed $\Pi_{\sigma}$ restricted to $\mathcal{F}_{\tau_{\sigma}}$ satisfies the market-local property. This proves the first part of the theorem. The second part follows from Theorem 3.10 and Theorem 3.11.

\section{A.4 Proofs of the results in Section 5.1}

Proof of Theorem 5.3. First assume that $g\left(t, z^{f}, z, \tilde{z}\right)-\theta_{t} z^{f}$ does not depend on $z^{f}$. We will prove that for every $\tau, \mathcal{E}_{\tau}^{g}$ is market-consistent, by using Proposition 3.3 (i) $\Rightarrow$ (iii). Without loss of generality assume that $\tau=0$. Let $H^{S} \in L^{\infty}\left(\overline{\mathcal{F}}_{T}^{S}\right)$. Denote by $W^{*, f}$ the Brownian motion under $\mathbb{Q}$, i.e., $W_{s}^{*, f}=W_{s}^{f}-\int_{0}^{s} \theta_{u} d u$, where the integral is defined componentwise. Since the financial market is complete there exists a predictable $n$-dimensional process $Z^{f} \in L^{2}\left(\overline{\mathcal{F}}^{S}, d \mathbb{Q} \times d u\right)$ such that $H^{S}=\int_{0}^{T} Z_{s}^{f} d W_{s}^{*, f}$. Define $Y_{s}=\mathbb{E}_{\mathcal{F}_{s}^{S}}^{\mathbb{Q}}\left[H^{S}\right]=\int_{0}^{s} Z_{u}^{f} d W_{u}^{*, f}$. Clearly, for every stopping time $\sigma$ we have that

$$
\mathbb{E}_{\overline{\mathcal{F}}_{\tau} S}\left[\int_{\sigma}^{T}\left|Z_{u}^{f}\right|^{2} d W_{u}^{*, f}\right] \leq 2\|Y\|_{S^{\infty}}^{2} \leq 2\|H\|_{\infty}^{2} .
$$

Predictable processes satisfying such a boundedness property are also called BMOs, see Kazamaki (1994). As $\theta$ is bounded it follows from Theorem 3.24 in Barrieu and El Karoui (2009) that $Z^{f}$ is a BMO under $\mathbb{P}$. In particular, $Z^{f} \in L^{2}\left(\overline{\mathcal{F}}^{S}, d \mathbb{P} \times d u\right)$. Now we get

$$
\begin{aligned}
d Y_{s} & =-g(s, 0,0,0) d s+Z_{s}^{f} d W_{s}^{*, f} \\
& =-\left(g\left(s, Z_{s}^{f}, 0,0\right)-\theta_{s} Z_{s}^{f}\right) d s+Z_{s}^{f} d W_{s}^{*, f}=-g\left(s, Z_{s}^{f}, 0,0\right) d s+Z_{s}^{f} d W_{s}^{f},
\end{aligned}
$$

where we used that $g(s, 0,0,0)=0$ in the first equation. In the second equation we applied that $g(s, 0,0,0)=g\left(s, z^{f}, 0,0\right)-\theta_{s} z^{f}$, as by assumption $g\left(s, z^{f}, 0,0\right)-\theta_{s} z^{f}$ does not depend on $z^{f}$. In the last equation we used the definition of $W^{*, f}$. This entails that $Y_{s}=\mathbb{E}_{\mathcal{\mathcal { F }}_{s}^{S}}^{\mathbb{Q}}[H]$ solves the BSDE with terminal condition $H$ and driver $g$. Therefore, indeed for every $H^{S}$ we have that $\mathbb{E}^{\mathbb{Q}}\left[H^{S}\right]=\mathcal{E}_{0}^{g}\left(H^{S}\right)$, and it follows from Proposition 3.3 (i) $\Rightarrow($ iii) (with $\mathcal{G}=\{\Omega, \emptyset\}$ ) that $\mathcal{E}_{0}^{g}$ is market-consistent. For general $\tau$ the argument is similar.

Now let us prove the other direction. For arbitrary $\bar{z}^{f} \in \mathbb{R}^{n}$ define

$$
\bar{g}\left(t, z^{f}, z, \tilde{z}\right):=g\left(t, z^{f}+\bar{z}^{f}, z, \tilde{z}\right)-\theta_{t} \bar{z}^{f}
$$

We need to show that $\bar{g}=g$. Let $\left(\mathcal{E}_{t}^{g}\left(H+\bar{z}^{f} W_{T}^{*, f}\right), Z^{f}, Z, \tilde{Z}\right)$ be the solution of the BSDE with terminal condition $H+\bar{z}^{f} W_{T}^{*, f}$ and driver function $g$. Note that the process $Y_{t}^{*}:=$ $\mathcal{E}_{t}^{g}\left(H+\bar{z}^{f} W_{T}^{*, f}\right)-\bar{z}^{f} W_{t}^{*, f}$ is equal to $H$ at time $T$. On the other hand we have

$$
\begin{aligned}
d Y_{t}^{*} & =-\left(g\left(t, Z_{t}^{f}, Z_{t}, \tilde{Z}_{t}\right)-\theta_{t} \bar{z}^{f}\right) d t+\left(Z_{t}^{f}-\bar{z}^{f}\right) d W_{t}^{f}+Z_{t} d W_{t}+\int_{\mathbb{R} \backslash\{0\}} \tilde{Z}_{t}(x) \tilde{N}(d t, d x) \\
& =-\bar{g}\left(t, Z_{\text {new }, t}^{f}, Z_{t}, \tilde{Z}_{t}\right) d t+Z_{n e w, t}^{f} d W_{t}^{f}+Z_{t} d W_{t}+\int_{\mathbb{R} \backslash\{0\}} \tilde{Z}_{t}(x) \tilde{N}(d t, d x),
\end{aligned}
$$


where $Z_{\text {new }}^{f}:=Z^{f}-\bar{z}^{f}$. Therefore, $Y^{*}$ solves the BSDE with terminal condition $H$ and driver $\bar{g}$. Hence, for every $t$ we have $\mathcal{E}_{t}^{g}\left(H+\bar{z}^{f} W_{T}^{*, f}\right)-\bar{z}^{f} W_{t}^{*, f}=Y_{t}^{*}=\mathcal{E}_{t}^{\bar{g}}(H)$. Denote by $\tilde{S}$ the vector of the discounted stock prices. By market-consistency we can conclude that for every $H \in L^{\infty}\left(\mathcal{F}_{T}\right)$

$$
\begin{aligned}
\mathcal{E}_{t}^{g}(H) & =\mathcal{E}_{t}^{g}\left(H+\int_{0}^{T} \bar{z}^{f} \tilde{\sigma}^{-1}\left(t, e^{r t} \tilde{S}_{t}\right) d \tilde{S}_{t}\right)-\int_{0}^{t} \bar{z}^{f} \tilde{\sigma}^{-1}\left(t, e^{r t} \tilde{S}_{t}\right) d \tilde{S}_{t} \\
& =\mathcal{E}_{t}^{g}\left(H+\bar{z}^{f} W_{T}^{*, f}\right)-\bar{z}^{f} W_{t}^{*, f}=\mathcal{E}_{t}^{\bar{g}}(H)
\end{aligned}
$$

for all $t$. Next choose $z^{f} \in \mathbb{R}^{n}, z \in \mathbb{R}^{d}$ and $\tilde{z} \in L^{2}(\nu(d x))$. Set

$$
H:=-\int_{0}^{T} g\left(s, z^{f}, z, \tilde{z}\right) d s+z^{f} W_{T}^{f}+z W_{T}+\int_{0}^{T} \int_{\mathbb{R} \backslash\{0\}} \tilde{z}(x) \tilde{N}(d s, d x) .
$$

Notice that $-\int_{0}^{t} g\left(s, z^{f}, z, \tilde{z}\right) d s+\int_{0}^{t} z^{f} d W_{s}^{f}+\int_{0}^{t} z d W_{s}+\int_{0}^{t} \int_{\mathbb{R} \backslash\{0\}} \tilde{z}(x) \tilde{N}(d s, d x)$ by definition is the solution of the BSDE with terminal condition $H$ and driver $g$. In particular, $\mathcal{E}_{0}^{g}(H)=0$. By (A.16) this yields $\mathcal{E}_{0}^{\bar{g}}(H)=\mathcal{E}_{0}^{g}(H)=0$ and

$$
\begin{aligned}
-\int_{0}^{t} g & \left(s, z^{f}, z, \tilde{z}\right) d s+\int_{0}^{t} z^{f} d W_{s}^{f}+\int_{0}^{t} z d W_{s}+\int_{0}^{t} \int_{\mathbb{R} \backslash\{0\}} \tilde{z}(x) \tilde{N}(d s, d x) \\
& =\mathcal{E}_{t}^{g}(H)=\mathcal{E}_{t}^{\bar{g}}(H) \\
& =\mathcal{E}_{0}^{\bar{g}}(H)-\int_{0}^{t} \bar{g}\left(s, Z_{s}^{f}, Z_{s}, \tilde{Z}_{s}\right) d s+\int_{0}^{t} Z_{s}^{f} d W_{s}^{f}+\int_{0}^{t} Z_{s} d W_{s}+\int_{0}^{t} \int_{\mathbb{R} \backslash\{0\}} \tilde{Z}_{s}(x) \tilde{N}(d s, d x) \\
& =-\int_{0}^{t} \bar{g}\left(s, Z_{s}^{f}, Z_{s}, \tilde{Z}_{s}\right) d s+\int_{0}^{t} Z_{s}^{f} d W_{s}^{f}+\int_{0}^{t} Z_{s} d W_{s}+\int_{0}^{t} \int_{\mathbb{R} \backslash\{0\}} \tilde{Z}_{s}(x) \tilde{N}(d s, d x),
\end{aligned}
$$

where $\left(Z^{f}, Z, \tilde{Z}\right)$ belong to the solution of the $\bar{g}$-expectation with terminal condition $H$. By the uniqueness of the decomposition of semi-martingales this entails that

$$
\begin{aligned}
\int_{0}^{t} z^{f} d W_{s}^{f} & +\int_{0}^{t} z d W_{s}+\int_{0}^{t} \int_{\mathbb{R} \backslash\{0\}} \tilde{z}(x) \tilde{N}(d s, d x) \\
& =\int_{0}^{t} Z_{s}^{f} d W_{s}^{f}+\int_{0}^{t} Z_{s} d W_{s}+\int_{0}^{t} \int_{\mathbb{R} \backslash\{0\}} \tilde{Z}_{s}(x) \tilde{N}(d s, d x),
\end{aligned}
$$

and

$$
\int_{0}^{t} g\left(s, z^{f}, z, \tilde{z}\right) d s=\int_{0}^{t} \bar{g}\left(s, Z_{s}^{f}, Z_{s}, \tilde{Z}_{s}\right) d s
$$

Taking for instance the quadratic covariation with respect to the components of $W^{f}, W$, and with respect to $\tilde{N}$ in (A.17) respectively, we may conclude that $Z_{t}^{f}=z^{f}, Z_{t}=z, d \mathbb{P} \times d t$ a.s., and $\tilde{Z}_{t}=\tilde{z}, \nu(d x) \times d \mathbb{P} \times d t$. But then (A.18) yields that for a.s. all $\omega$

$$
\int_{0}^{t} g\left(s, z^{f}, z, \tilde{z}\right) d s=\int_{0}^{t} \bar{g}\left(s, z^{f}, z, \tilde{z}\right) d s, \text { for all } t \in[0, T]
$$

and therefore $g\left(t, z^{f}, z, \tilde{z}\right)=\bar{g}\left(t, z^{f}, z, \tilde{z}\right)$ for a.s. all $\omega$ for Lesbegue a.s. all $t$. 


\section{A.5 Proofs of the results in Section 5.2}

Proof of Proposition 5.5. First of all note that since $\frac{d \mathbb{Q}^{h}}{d \mathbb{P}}$ is $\sigma\left(W_{t}^{f, *} \mid 0 \leq t \leq T\right)$-measurable, we have that $W$ and $\tilde{N}$ have the same joint distribution under $\mathbb{Q}^{h}$ as under $\mathbb{P}$ (since they are independent of $\left.W^{f, *}\right)$.

By well known projection results, there exists adapted $Z_{i h}^{h, f}: \Omega \rightarrow \mathbb{R}^{n}, Z_{i h}^{h}: \Omega \rightarrow \mathbb{R}^{d}$, measurable with respect to $\mathcal{F}_{i h}, \tilde{Z}_{i h}^{h}: \Omega \times \mathbb{R} \backslash\{0\} \rightarrow \mathbb{R}$, measurable with respect to $\mathcal{F}_{i h} \otimes \mathcal{B}(\mathbb{R} \backslash$ $\{0\}$ ), and a real-valued $\mathbb{Q}^{h}$-martingale $\left(L_{i h}^{h}\right)_{i}$ which is orthogonal (under $\mathbb{Q}^{h}$ ) to $W_{i h}^{f, *}, W_{i h}$, and $\tilde{N}((0, i h], d x)$, such that

$$
\begin{aligned}
\Pi_{(i+1) h}(H)= & \mathbb{E}_{\mathcal{F}_{i h}}^{\mathbb{F}_{i h}^{h}}\left[\Pi_{(i+1) h}(H)\right]+Z_{i h}^{h, f} \Delta W_{(i+1) h}^{f, *}+Z_{i h}^{h} \Delta W_{(i+1) h} \\
& +\int_{\mathbb{R} \backslash\{0\}} \tilde{Z}_{i h}^{h}(x) \tilde{N}((i h,(i+1) h], d x)+\Delta L_{(i+1) h}^{h} .
\end{aligned}
$$

For the sake of simplicity we will omit the superscript $h$ for the $Z^{h, f}, Z^{h}$, and $\tilde{Z}^{h}$ in the sequel.

It follows that

$$
\begin{aligned}
\Delta & \Pi_{(i+1) h}(H) \\
= & \Pi_{(i+1) h}(H)-\Pi_{i h}(H) \\
= & \Pi_{(i+1) h}(H)-\Pi_{i h,(i+1) h}^{v}\left(\Pi_{(i+1) h}(H)\right) \\
= & Z_{i h}^{f} \Delta W_{(i+1) h}^{f, *}+Z_{i h} \Delta W_{(i+1) h}+\int_{\mathbb{R} \backslash\{0\}} \tilde{Z}_{i h}(x) \tilde{N}((i h,(i+1) h], d x)+\Delta L_{(i+1) h} \\
& -\Pi_{i h}^{v}\left(Z_{j h}^{f} \Delta W_{(i+1) h}^{f, *}+Z_{i h} \Delta W_{(i+1) h}+\int_{\mathbb{R} \backslash\{0\}} \tilde{Z}_{i h}(x) \tilde{N}((i h,(i+1) h], d x)+\Delta L_{(i+1) h}\right) \\
= & Z_{j h}^{f} \Delta W_{(i+1) h}^{f, *}+Z_{i h} \Delta W_{(i+1) h}+\int_{\mathbb{R} \backslash\{0\}} \tilde{Z}_{i h}(x) \tilde{N}((i h,(i+1) h], d x)+\Delta L_{(i+1) h} \\
& -\Pi_{i h,(i+1) h}^{v}\left(Z_{i h} \Delta W_{(i+1) h}+\int_{\mathbb{R} \backslash\{0\}} \tilde{Z}_{i h}(x) \tilde{N}((i h,(i+1) h], d x)+\Delta L_{(i+1) h}\right) \\
\stackrel{!}{=} & Z_{j h}^{f} \Delta W_{(i+1) h}^{f}+Z_{i h} \Delta W_{(i+1) h}+\int_{\mathbb{R} \backslash\{0\}} \tilde{Z}_{i h}(x) \tilde{N}((i h,(i+1) h], d x)+\Delta L_{(i+1) h} \\
& -\left[\theta_{i h} Z_{i h}^{f}+\frac{\alpha}{2}\left|Z_{i h}\right|^{2}+\frac{\alpha}{2} \int_{\mathbb{R} \backslash\{0\}}\left|\tilde{Z}_{i h}(x)\right|^{2} \nu(d x)\right] h \\
& -\frac{\alpha}{2} \mathbb{E}_{\mathcal{Q}_{i h}^{h}}^{\mathbb{Q}_{i n}}\left[\left(\Delta L_{(i+1) h}-\mathbb{E}_{\mathcal{F}_{(i+1) h}^{S h}}\left[\Delta L_{(i+1) h}\right]\right)^{2}\right],
\end{aligned}
$$

where we have used (5.1) in the third equation. Furthermore, we applied cash invariance in the third and market-consistency in the fourth equation. To see that the last equation holds denote by $\operatorname{Cov}_{\mathbb{P}_{\mathcal{F}}^{S h}(i+1) h}\left(X_{1}, X_{2}\right)$ the covariance of $X_{1}$ and $X_{2}$ with respect to $\mathbb{P}_{\mathcal{F}_{(i+1) h}^{S h}}$. Since all random variables are $\mathcal{F}_{(i+1) h}$-measurable we may assume that $\frac{d \mathbb{Q}^{h}}{d \mathbb{P}}$ is $\mathcal{F}_{(i+1) h}^{S^{h}}$-measurable. 
It is

$$
\begin{aligned}
& \Pi_{i h,(i+1) h}^{v}\left(Z_{i h} \Delta W_{(i+1) h}+\int_{\mathbb{R} \backslash\{0\}} \tilde{Z}_{i h}(x) \tilde{N}((i h,(i+1) h], d x)+\Delta L_{(i+1) h}\right) \\
& =\frac{\alpha}{2} \mathbb{E}_{\mathcal{F}_{i h}}^{\mathbb{Q}^{h}}\left[\operatorname{Var}_{\mathcal{F}_{(i+1) h}^{S h}}\left(Z_{i h} \Delta W_{(i+1) h}+\int_{\mathbb{R} \backslash\{0\}} \tilde{Z}_{i h}(x) \tilde{N}((i h,(i+1) h], d x)+\Delta L_{(i+1) h}\right)\right] \\
& =\frac{\alpha}{2}\left(h\left|Z_{i h}\right|^{2}+h \int_{\mathbb{R} \backslash\{0\}}\left|\tilde{Z}_{i h}(x)\right|^{2} \nu(d x)+\mathbb{E}_{\mathcal{F}_{i h}}^{\mathbb{Q}^{h}}\left[\left(\Delta L_{(i+1) h}-\mathbb{E}_{\mathcal{F}_{(i+1) h}^{S h}}\left[\Delta L_{(i+1) h}\right]\right)^{2}\right]\right. \\
& +2 \sum_{j=1}^{d} Z_{i h}^{j} \mathbb{E}_{\mathcal{F}_{i h}}^{\mathbb{Q}^{h}}\left[\operatorname{Cov}_{\mathbb{P}_{\mathcal{F}_{(i+1) h}^{S h}}}\left(\Delta W_{(i+1) h}^{j}, \Delta L_{(i+1) h}\right)\right] \\
& +2 \mathbb{E}_{\mathcal{F}_{i h}}^{\mathbb{Q}^{h}}\left[\operatorname{Cov}_{\mathbb{P}_{\mathcal{F}_{(i+1) h}^{S h}}}\left(\int_{\mathbb{R} \backslash\{0\}} \tilde{Z}_{i h}(x) \tilde{N}((i h,(i+1) h], d x), \Delta L_{(i+1) h}\right)\right] \\
& \left.+2 \sum_{j=1}^{d} Z_{i h}^{j} \mathbb{E}_{\mathcal{F}_{i h}^{h}}^{\mathbb{Q}_{i h}}\left[\operatorname{Cov}_{\mathbb{P}_{(i+1) h}^{S h}}\left(\Delta W_{(i+1) h}^{j}, \int_{\mathbb{R} \backslash\{0\}} \tilde{Z}_{i h}(x) \tilde{N}((i h,(i+1) h], d x)\right)\right]\right),
\end{aligned}
$$

where we have used that $W$ and $\tilde{N}$ are independent of $S^{h}$ and that $\frac{d \mathbb{Q}^{h}}{d \mathbb{P}}$ is $\mathcal{F}_{(i+1) h^{-m e a s u r a b l e}}^{S^{h}}$

Hence, to prove (A.19) the only thing what remains left is to show that the the covariance terms in (A.20) are zero. By Lemma A.8 (with $\hat{\mathbb{P}}=\mathbb{Q}^{h}$ and $\overline{\mathbb{P}}=\mathbb{P}$ ) we get for $j=1, \ldots, d$

$$
\mathbb{E}_{\mathcal{F}_{(i+1) h}^{S h}}^{\mathbb{Q}^{h}}\left[\Delta W_{(i+1) h}^{j}\right]=\mathbb{E}_{\mathcal{F}_{(i+1) h}^{S h}}\left[\Delta W_{(i+1) h}^{j}\right]=\mathbb{E}_{\mathcal{F}_{i h}}\left[\Delta W_{(i+1) h}^{j}\right]=0,
$$

where we used the independence of $S^{h}$ and $W$ in the second equation. Hence, since $L_{(i+1) h}$ and $\Delta W_{(i+1) h}$ are orthogonal under $\mathbb{Q}_{\mathcal{F}_{i h}}^{h}$, we may conclude that

$$
\begin{aligned}
\mathbb{E}_{\mathcal{F}_{i h}}^{\mathbb{Q}^{h}}\left[\operatorname{Cov}_{\left.\mathbb{P}_{\mathcal{F}_{(i+1) h}^{S h}}\left(\Delta W_{(i+1) h}^{j}, \Delta L_{(i+1) h}\right)\right]}\right. & =\mathbb{E}_{\mathcal{F}_{i h}}^{\mathbb{Q}^{h}}\left[\Delta W_{(i+1) h}^{j} \Delta L_{(i+1) h}\right] \\
& =\operatorname{Cov}_{\mathbb{Q}_{\mathcal{F}_{i h}}^{h}}\left(\Delta W_{(i+1) h}^{j}, \Delta L_{(i+1) h}\right)=0 .
\end{aligned}
$$

Similarly, it may be seen that the second and third covariance terms in (A.19) are zero. Thus, (A.19) is proved. From (A.19) we may finally conclude

$$
\begin{aligned}
\Pi_{T}( & H)-\Pi_{i h}(H) \\
= & \sum_{j=i}^{T / h-1} \Delta \Pi_{(j+1) h}(H) \\
= & \sum_{j=i}^{T / h-1}\left(Z_{j h}^{f} \Delta W_{(j+1) h}^{f}+Z_{j h} \Delta W_{(j+1) h}+\int_{\mathbb{R} \backslash\{0\}} \tilde{Z}_{j h}(x) \tilde{N}((j h,(j+1) h], d x)+\Delta L_{(j+1) h}\right) \\
& -\sum_{j=i}^{T / h-1}\left[\theta_{j h} Z_{j h}^{f}+\frac{\alpha}{2}\left|Z_{j h}\right|^{2}+\frac{\alpha}{2} \int_{\mathbb{R} \backslash\{0\}}\left|\tilde{Z}_{j h}(x)\right|^{2} \nu(d x)\right] h \\
& +\frac{\alpha}{2} \mathbb{E}_{\mathcal{Q}_{j h}^{h}}\left[\left(\Delta L_{(j+1) h}-\mathbb{E}_{\mathcal{F}_{(j+1) h}^{S h}}\left[\Delta L_{(j+1) h}\right]\right)^{2}\right] .
\end{aligned}
$$


Since by construction $\Pi_{T}(H)=H$ the proposition is proved.

\section{References}

Artzner, P., Delbaen, F., Eber, J., and Heath, D. (1999). Coherent measures of risk. Mathematical Finance, 9(3):203-228.

Artzner, P., Delbaen, F., Eber, J., Heath, D., and Ku, H. (2007). Coherent multiperiod risk adjusted values and bellmans principle. Annals of Operations Research, 152(1):5-22.

Artzner, P. and Eisele, K. (2010). Supervisory accounting: comparison between solvency 2 and coherent risk measures. In Actuarial and Financial Mathematics Conference: Interplay between Finance and Insurance, pages 3-15.

Barles, G., Buckdahn, R., and Pardoux, E. (1997). Backward stochastic differential equations and integral-partial differential equations. Stochastics and Stochastics Reports, 60(1):57-84.

Barrieu, P. and El Karoui, N. (2005). Inf-convolution of risk measures and optimal risk transfer. Finance and Stochastics, 9(2):269-298.

Barrieu, P. and El Karoui, N. (2009). Pricing, hedging, and designing derivatives with risk measures. In Carmona (2009).

Bion-Nadal, J. (2008). Dynamic risk measures: time consistency and risk measures from bmo martingales. Finance and Stochastics, 12(2):219-244.

Bion-Nadal, J. (2009). Time consistent dynamic risk processes. Stochastic Processes and their Applications, 119(2):633-654.

Björk, T. and Slinko, I. (2006). Towards a general theory of good-deal bounds. Review of Finance, 10(2):221-260.

Black, F. and Scholes, M. (1973). The pricing of options and corporate liabilities. Journal of Political Economy, 81:637-659.

Bühlmann, H. (1970). Mathematical Methods in Risk Theory. Springer Verlag, Berlin.

Carmona, R. (2009). Indifference Pricing: Theory and Applications. Princeton Univ Press.

Černý, A. and Hodges, S. (2002). The theory of good-deal pricing in financial markets. In H. Geman, D. Madan, S. R. P. and Vorst, T., editors, Mathematical Finance, Bachelier Congress 2000, pages 172-202. Springer.

Chen, Z. and Epstein, L. (2002). Ambiguity, risk, and asset returns in continuous time. Econometrica, 70(4):1403-1443.

Cheridito, P., Delbaen, F., and Kupper, M. (2006). Dynamic monetary risk measures for bounded discrete-time processes. Electronic Journal of Probability, 11(3):57-106.

Cheridito, P. and Kupper, M. (2009). Recursiveness of indifference prices and translationinvariant preferences. Mathematics and Financial Economics, 2(3):173-188. 
Cheridito, P. and Kupper, M. (2011). Composition of time-consistent dynamic monetary risk measures in discrete time. International Journal of Theoretical and Applied Finance, 14(1):137-162.

Cheridito, P. and Stadje, M. (2010). Bs $\delta$ es and bsdes with non-lipschitz drivers: comparison, convergence and robustness. To appear in: Bernoulli.

Cochrane, J. H. and Saá-Requejo, J. (2000). Beyond arbitrage: good-deal asset price bounds in incomplete markets. Journal of Political Economy, 108(1):79-119.

Cont, R. (2006). Model uncertainty and its impact on derivative instruments. Mathematical Finance, 16(6):519-542.

Coquet, F., Hu, Y., Memin, J., and Peng, S. (2002). Filtration-consistent nonlinear expectations and related g-expectations. Probability Theory and Related Fields, 123(1):1-27.

Cvitanic, J. and Karatzas, I. (1992). Convex duality in constrained portfolio optimization. Annals of Applied Probability, 2(4):767-818.

Delbaen, F. (2006). The structure of $m$-stable sets and in particular of the set of risk neutral measures. In Émery, M. and Yor, M., editors, Séminaire de Probabilités (Vol. XXXIX), Lecture Notes in Mathematics (Vol. 1874), pages 215-258. Springer.

Delbaen, F. and Schachermayer, W. (1994). A general version of the fundamental theorem of asset pricing. Mathematische Annalen, 300(3):463-520.

Delbaen, F. and Schachermayer, W. (1996). The variance-optimal martingale measure for continuous processes. Bernoulli, 2(1):81-105.

Deprez, O. and Gerber, H. (1985). On convex principles of premium calculation. Insurance: Mathematics and Economics, 4(3):179-189.

Duffie, D. and Epstein, L. (1992). Stochastic differential utility. Econometrica, 60(2):353-394.

El Karoui, N., Peng, S., and Quenez, M. (1997). Backward stochastic differential equations in finance. Mathematical Finance, 7(1):1-71.

El Karoui, N. and Ravanelli, C. (2009). Cash subadditive risk measures and interest rate ambiguity. Mathematical Finance, 19(4):561-590.

Filipovic, D. and Kupper, M. (2008). Equilibrium prices for monetary utility functions. International Journal of Theoretical and Applied Finance, 11(3):325-343.

Föllmer, H. and Schied, A. (2002). Convex measures of risk and trading constraints. Finance and Stochastics, 6(4):429-447.

Föllmer, H. and Schied, A. (2004). Stochastic Finance. 2nd edition, de Gruyter.

Föllmer, H. and Schweizer, M. (1989). Hedging by sequential regression: An introduction to the mathematics of option trading. ASTIN Bulletin, 18(2):147-160.

Frittelli, M. and Gianin, E. (2004). Dynamic convex risk measures. Risk Measures for the 21st Century, pages 227-248. 
Frittelli, M. and Rosazza Gianin, E. (2002). Putting order in risk measures. Journal of Banking E Finance, 26(7):1473-1486.

Gerber, H. (1979). An Introduction to Mathematical Risk Theory. Irwin Homewood.

Hamadène, S. and Jeanblanc, M. (2007). On the starting and stopping problem: application in reversible investments. Mathematics of Operations Research, 32(1):182.

Hansen, L. and Sargent, T. (2001). Robust control and model uncertainty. American Economic Review, 91(2):60-66.

Hansen, L. and Sargent, T. (2007). Robustness. Princeton University Press Princeton, NJ.

Hardy, M. and Wirch, J. (2005). The iterated CTE-a dynamic risk measure. North American Actuarial Journal, 8(4):62-75.

Henderson, V. (2002). Valuation of claims on nontraded assets using utility maximization. Mathematical Finance, 12(4):351-373.

Hobson, D. (2004). Stochastic volatility models, correlation, and the $q$-optimal measure. Mathematical Finance, 14(4):537-556.

Hodges, S. and Neuberger, A. (1989). Optimal replication of contingent claims under transaction costs. Review of Futures Markets, 8(2):222-239.

Jacod, J. and Protter, P. (2010). Risk-neutral compatibility with option prices. Finance and Stochastics, 14(2):285-315.

Jacod, J. and Shiryaev, A. (1987). Limit Theorems for Stochastic Processes, volume 2003. Springer.

Jobert, A. and Rogers, L. (2008). Valuations and dynamic convex risk measures. Mathematical Finance, 18(1):1-22.

Jouini, E., Schachermayer, W., and Touzi, N. (2008). Optimal risk sharing for law invariant monetary utility functions. Mathematical Finance, 18(2):269-292.

Kaas, R., Goovaerts, M., Dhaene, J., and Denuit, M. (2008). Modern Actuarial Risk Theory: Using R. Springer.

Kazamaki, N. (1994). Continuous Exponential Martingales and BMO. Volume 1579 of Lecture Notes in Mathematics, Springer-Verlag, Berlin.

Klöppel, S. and Schweizer, M. (2007). Dynamic indifference valuation via convex risk measures. Mathematical Finance, 17(4):599-627.

Knispel, T., Stahl, G., and Weber, S. (2011). From the equivalence principle to market consistent valuation. Preprint.

Kramkov, D. and Schachermayer, W. (1999). The asymptotic elasticity of utility functions and optimal investment in incomplete markets. Annals of Applied Probability, 9(3):904-950. 
Kupper, M., Cheridito, P., and Filipovic, D. (2008). Dynamic risk measures, valuations and optimal dividends for insurance. In Mini-Workshop: Mathematics of Solvency. Mathematisches Forschungsinstitut Oberwolfach.

Lazrak, A. and Quenez, M. (2003). A generalized stochastic differential utility. Mathematics of Operations Research, 28(1):154-180.

Maccheroni, F., Marinacci, M., and Rustichini, A. (2006). Dynamic variational preferences. Journal of Economic Theory, 128(1):4-44.

Madan, D., Pistorius, M., and Schoutens, W. (2010). The valuation of structured products using markov chain models. Preprint.

Malamud, S., Trubowitz, E., and Wüthrich, M. (2008). Market consistent pricing of insurance products. ASTIN Bulletin, 38(2):483-526.

Møller, T. (2002). On valuation and risk management at the interface of insurance and finance. British Actuarial Journal, 8(4):787-828.

Monoyios, M. (2006). Characterisation of optimal dual measures via distortion. Decisions in Economics and Finance, 29(2):95-119.

Morlais, M. (2010). A new existence result for quadratic bsdes with jumps with application to the utility maximization problem. Stochastic Processes and their Applications, 120(10):19661995.

Musiela, M. and Zariphopoulou, T. (2004a). An example of indifference prices under exponential preferences. Finance and Stochastics, 8(2):229-239.

Musiela, M. and Zariphopoulou, T. (2004b). A valuation algorithm for indifference prices in incomplete markets. Finance and Stochastics, 8(3):399-414.

Peng, S. (2004). Filtration consistent nonlinear expectations and evaluations of contingent claims. Acta Mathematicae Applicatae Sinica (English Series), 20(2):191-214.

Porchet, A., Touzi, N., and Warin, X. (2009). Valuation of a power plant under production constraints and market incompleteness. Mathematical Methods of Operations Research, $70(1): 47-75$.

Riedel, F. (2004). Dynamic coherent risk measures. Stochastic Processes and their Applications, 112(2):185-200.

Rogers, L. (2001). Duality in constrained optimal investment and consumption problems: a synthesis. In Workshop on Financial Mathematics and Econometrics held in Montreal. Springer.

Roorda, B., Schumacher, J., and Engwerda, J. (2005). Coherent acceptability measures in multiperiod models. Mathematical Finance, 15(4):589-612.

Rosazza Gianin, E. (2006). Risk measures via g-expectations. Insurance Mathematics and Economics, 39(1):19-34. 
Royer, M. (2006). Backward stochastic differential equations with jumps and related non-linear expectations. Stochastic processes and their Applications, 116(10):1358-1376.

Ruszczynski, A. and Shapiro, A. (2006). Conditional risk mappings. Mathematics of Operations Research, 31(3):544-561.

Schweizer, M. (1995). On the minimal martingale measure and the Föllmer-Schweizer decomposition. Stochastic Analysis and Applications, 13(5):573-600.

Schweizer, M. (2010). Mean-variance hedging and mean-variance portfolio selection. Encyclopedia of Quantitative Finance. Wiley, Chichester.

Stadje, M. (2010). Extending dynamic convex risk measures from discrete time to continuous time: A convergence approach. Insurance: Mathematics and Economics, 57(3):391-404.

Toussaint, A. and Sircar, R. (2011). A framework for dynamic hedging under convex risk measures. In Seminar on Stochastic Analysis, Random Fields and Applications VI, pages 429-451. Springer.

Wüthrich, M., Bühlmann, H., and Furrer, H. (2008). Market-Consistent Actuarial Valuation. European Actuarial Academy, (EAA), Series, Springer, Heidelberg.

Young, V. R. and Zariphopoulou, T. (2002). Pricing dynamic insurance risks using the principle of equivalent utility. Scandinavian Actuarial Journal, 2002(4):246-279.

Zălinescu, C. (2002). Convex Analysis in General Vector Spaces. World Scientific Pub Co Inc. 